\title{
Multilevel Methods for Uncertainty Quantification of Elliptic PDEs with Random Anisotropic Diffusion
}

\author{
Helmut Harbrecht • Marc Schmidlin
}

Received: date / Accepted: date

\begin{abstract}
We consider elliptic diffusion problems with a random anisotropic diffusion coefficient, where, in a notable direction given by a random vector field, the diffusion strength differs from the diffusion strength perpendicular to this notable direction. The Karhunen-Loève expansion then yields a parametrisation of the random vector field and, therefore, also of the solution of the elliptic diffusion problem. We show that, given regularity of the elliptic diffusion problem, the decay of the Karhunen-Loève expansion entirely determines the regularity of the solution's dependence on the random parameter, also when considering this higher spatial regularity. This result then implies that multilevel quadrature methods may be used to lessen the computation complexity when approximating quantities of interest, like the solution's mean or its second moment, while still yielding the expected rates of convergence. Numerical examples in three spatial dimensions are provided to validate the presented theory.
\end{abstract}

Keywords Uncertainty quantification · Anisotropic diffusion · Regularity estimates · Multilevel methods

Mathematics Subject Classification (2000) 35R60 65 N30 60 H35

\section{Introduction}

The numerical approximation of quantities of interest, such as expectation, variance, or more general output functionals, of the solution of a diffusion problem with a scalar random diffusion coefficient with multilevel collocation or multilevel quadrature methods has been considered previously, see e.g. [2, $8,13,14,20,23,28$,

Funding: The work of the authors was supported by the Swiss National Science Foundation (SNSF) through the project "Multilevel Methods and Uncertainty Quantification in Cardiac Electrophysiology" (grant 205321_169599).

Helmut Harbrecht and Marc Schmidlin

Departement Mathematik und Informatik, Universität Basel, Spiegelgasse 1, 4051 Basel, Switzerland

E-mail: \{helmut.harbrecht, marc.schmidlin\}@unibas.ch 
$35]$ and the references therein; in this isotropic case, the mixed smoothness required for the use of such multilevel methods has been provided in [9] for uniformly elliptic diffusion coefficients and in [26] for log-normally distributed diffusion coefficients. However, in simulations of certain diffusion phenomena in science and engineering, the diffusion that needs to be modeled may not necessarily be isotropic. One specific application we have in mind here stems from cardiac electrophysiology, where the electrical activation of the human heart is considered. It is known that the fibrous structure of the heart plays a major role when considering the electrical and mechanical properties of the heart. And while the fibres have a complex and generally well-organised structure, see e.g. $[11,31,32,33]$, the exact fibre orientation may vary between individuals and also over time in an individual, for example due to the presence of scaring of the heart.

More generally, we wish to be able to model diffusion in a fibrous media, where fibre direction and diffusion strength in fibre direction are subject to uncertainty. For this setting, the following random anisotropic diffusion coefficient was defined in $[24]$ :

$$
\mathbf{A}[\omega]:=a \mathbf{I}+\left(\|\mathbf{V}[\omega]\|_{2}-a\right) \frac{\mathbf{V}[\omega] \mathbf{V}^{\top}[\omega]}{\mathbf{V}^{\top}[\omega] \mathbf{V}[\omega]},
$$

where $a$ is a given value, $\mathbf{I}$ is the identity matrix of $\mathbb{R}^{d \times d}$ and $\mathbf{V}$ is a random $\mathbb{R}^{d}$-valued field, over a given spatial domain $D \subset \mathbb{R}^{d}$ and a given probability space $(\Omega, \mathcal{F}, \mathbb{P})$. The fibre direction is hence given by $\mathbf{V} /\|\mathbf{V}\|_{2}$ with the diffusion strength in the fibre direction being $\|\mathbf{V}\|_{2}$ and the diffusion strength perpendicular to the fibre direction is defined by $a$. While we only consider this model hereafter, the techniques we use may also be applied straightforwardly to other models of random anisotropic diffusion coefficients, such as, for example, the following model in three spatial dimensions:

$$
\begin{aligned}
& \mathbf{A}[\omega]:=\left[\begin{array}{lll}
\mid & \mid & \mid \\
\mathbf{f} & \mathbf{t} & \mathbf{s} \\
\mid & \mid & \mid
\end{array}\right]\left[\begin{array}{ccc}
\cos \alpha_{3}[\omega] & -\sin \alpha_{3}[\omega] & 0 \\
\sin \alpha_{3}[\omega] & \cos \alpha_{3}[\omega] & 0 \\
0 & 0 & 1
\end{array}\right]\left[\begin{array}{ccc}
1 & 0 & 0 \\
0 & \cos \alpha_{2}[\omega] & \sin \alpha_{2}[\omega] \\
0 & -\sin \alpha_{2}[\omega] & \cos \alpha_{2}[\omega]
\end{array}\right] \\
& {\left[\begin{array}{ccc}
\cos \alpha_{1}[\omega] & 0 & \sin \alpha_{1}[\omega] \\
0 & 1 & 0 \\
-\sin \alpha_{1}[\omega] & 0 & \cos \alpha_{1}[\omega]
\end{array}\right]\left[\begin{array}{ccc}
a_{\mathbf{f}}[\omega] & 0 & 0 \\
0 & a_{\mathbf{t}}[\omega] & 0 \\
0 & 0 & a_{\mathbf{s}}[\omega]
\end{array}\right]\left[\begin{array}{ccc}
\cos \alpha_{1}[\omega] & 0 & \sin \alpha_{1}[\omega] \\
0 & 1 & 0 \\
-\sin \alpha_{1}[\omega] & 0 & \cos \alpha_{1}[\omega]
\end{array}\right]^{\top}}
\end{aligned}
$$

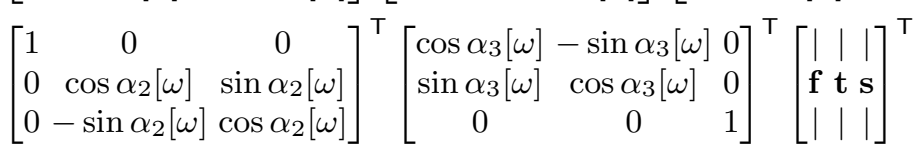

Here, $\mathbf{f}, \mathbf{t}$ and $\mathbf{s}$ are vector fields describing the fibre, the transverse sheet and the sheet normal directions in the heart, which at each point in $D$ yields an orthonormal basis. These vector fields could, for example, be derived from measurements or be generated by an algorithm such as the Laplace-Dirichlet Rule-Based algorithm described in [3]. Note that, in this model, the diffusion strengths are random fields $a_{\mathbf{f}}, a_{\mathbf{t}}$ and $a_{\mathbf{s}}$, and the fibre and sheet directions are locally angularily perturbed by random fields $\alpha_{1}, \alpha_{2}$ and $\alpha_{3}$.

We shall consider the second order diffusion problem with this uncertain diffusion coefficient $\mathbf{A}$ given by

$$
\text { for almost every } \omega \in \Omega:\left\{\begin{aligned}
-\operatorname{div}_{\mathbf{x}}\left(\mathbf{A}[\omega] \nabla_{\mathbf{x}} u[\omega]\right)=f & \text { in } D, \\
u[\omega]=0 & \text { on } \partial D,
\end{aligned}\right.
$$


with the known function $f$ as a source. The result of this article is then as follows. Having spatial $H^{s}$-regularity of the underlying diffusion problem, given by sufficient smoothness of the right hand $f$ side and the domain $D$, then the random solution $u$ admits analytic regularity with respect to the stochastic parameter also in the $H^{s}(D)$-norm provided that the random vector-valued field offers enough spatial regularity. This mixed regularity is the essential ingredient in order to apply multilevel collocation or multilevel quadrature methods without deteriorating the rate of convergence, see [20] for instance.

The rest of the article is organised as follows: In Section 2, we provide basic definitions and notation for the functional analytic framework to be able to state and then also reformulate the model problem, by using the Karhunen-Loève expansion of the diffusion describing random vector-valued field $\mathbf{V}$, into its stochastically parametric and spatially weak formulation. Section 3 then deals with the regularity of the solution of the stochastically parametric and spatially weak formulation of the model problem with respect to the stochastic parameter and some given higher spatial regularity in the model problem. We then use the fact that the higher spatial regularity can be kept, when considering the regularity of the solution with respect to the stochastic parameter, to arrive at convergence rates when considering multilevel quadrature, such as multilevel quasi-Monte Carlo quadrature, to approximate the solution's mean and second moment. Numerical examples are provided in Section 4 as validation; specifically we use multilevel quasi-Monte Carlo quadrature to approximate the solution's mean and second moment in a setting with three spatial dimensions. Lastly, we give our conclusions in Section 5.

\section{Notation and model problem}

\subsection{Notation and precursory remarks}

We denote the natural numbers including 0 by $\mathbb{N}$ and excluding 0 by $\mathbb{N}^{*}$.

For a sequence of natural numbers, $\boldsymbol{\alpha}=\left\{\alpha_{n}\right\}_{n \in \mathbb{N}^{*}} \in \mathbb{N}^{\mathbb{N}^{*}}$, we as usual define the support of the sequence as

$$
\operatorname{supp} \boldsymbol{\alpha}=\left\{n \in \mathbb{N}^{*} \mid \alpha_{n} \neq 0\right\} .
$$

If $\operatorname{supp} \boldsymbol{\alpha}$ is of finite cardinality, we say that $\boldsymbol{\alpha}$ is finitely supported. The set of finitely supported sequences of natural numbers is then denoted by $\mathbb{N}_{f}^{\mathbb{N}^{*}}$, while we will refer to its elements as multi-indices. For all $m \in \mathbb{N}^{*}$ we will identify the elements $\boldsymbol{\alpha}=\left(\alpha_{1}, \ldots, \alpha_{m}\right) \in \mathbb{N}^{m}$ with their extension by zero into $\mathbb{N}_{f}^{\mathbb{N}^{*}}$, that is $\boldsymbol{\alpha}=\left(\alpha_{1}, \ldots, \alpha_{m}, 0, \ldots\right)$, so that all notations defined below for elements of $\mathbb{N}_{f}^{\mathbb{N}^{*}}$ also carry over to the elements of $\mathbb{N}^{m}$; we then also refer to elements of $\mathbb{N}^{m}$ as multi-indices.

For multi-indices $\boldsymbol{\alpha}=\left\{\alpha_{n}\right\}_{n \in \mathbb{N}^{*}}, \boldsymbol{\beta}=\left\{\beta_{n}\right\}_{n \in \mathbb{N}^{*}} \in \mathbb{N}_{f}^{\mathbb{N}^{*}}$ and a sequence of real numbers $\gamma=\left\{\gamma_{n}\right\}_{n \in \mathbb{N}^{*}} \in \mathbb{R}^{\mathbb{N}^{*}}$, we use the following common notations:

$$
\begin{array}{rlrl}
|\boldsymbol{\alpha}| & :=\sum_{n \in \operatorname{supp} \boldsymbol{\alpha}} \alpha_{n}, & \boldsymbol{\alpha} !:=\prod_{n \in \operatorname{supp} \boldsymbol{\alpha}} \alpha_{n} !, \\
\left(\begin{array}{c}
\boldsymbol{\alpha} \\
\boldsymbol{\beta}
\end{array}\right):=\prod_{n \in \operatorname{supp} \boldsymbol{\alpha} \cup \operatorname{supp} \boldsymbol{\beta}}\left(\begin{array}{c}
\alpha_{n} \\
\beta_{n}
\end{array}\right), & \gamma^{\boldsymbol{\alpha}}:=\prod_{n \in \operatorname{supp} \boldsymbol{\alpha}} \gamma_{n}^{\alpha_{n}} .
\end{array}
$$


Furthermore, we say that $\boldsymbol{\alpha} \leq \boldsymbol{\beta}$ holds, when $\alpha_{j} \leq \beta_{j}$ holds for all $j \in \operatorname{supp} \boldsymbol{\alpha} \cup$ $\operatorname{supp} \boldsymbol{\beta}$, and $\boldsymbol{\alpha}<\boldsymbol{\beta}$, when $\boldsymbol{\alpha} \leq \boldsymbol{\beta}$ and $\boldsymbol{\alpha} \neq \boldsymbol{\beta}$ hold.

Subsequently, we will always equip $\mathbb{R}^{m}$ with the norm $\|\cdot\|_{2}$ induced by the canonical inner product $\langle\cdot, \cdot\rangle$ and $\mathbb{R}^{m \times m}$ with the induced norm $\|\cdot\|_{2}$. Then, for $\mathbf{v}, \mathbf{w} \in \mathbb{R}^{d}$, the Cauchy-Schwartz inequality gives us

$$
\left|\mathbf{v}^{\top} \mathbf{w}\right|=|\langle\mathbf{v}, \mathbf{w}\rangle| \leq\|\mathbf{v}\|_{2}\|\mathbf{w}\|_{2},
$$

and we also have, by straightforward computation, that

$$
\left\|\mathbf{v} \mathbf{w}^{\top}\right\|_{2}=\|\mathbf{v}\|_{2}\|\mathbf{w}\|_{2} .
$$

Moreover, when considering $\mathbb{R}^{m}$ itself or an open domain $\mathcal{D} \subset \mathbb{R}^{m}$ as a measure space we always equip it with the Lebesgue measure. Similarly, we always equip $\mathbb{N}$ and $\mathbb{N}^{*}$ with the counting measure when considering them as measure spaces.

Let $\mathcal{X}, \mathcal{X}_{1}, \ldots, \mathcal{X}_{r}$ and $\mathcal{Y}$ be Banach spaces, then we denote the Banach space of bounded, linear maps from $\mathcal{X}$ to $\mathcal{Y}$ as $\mathcal{B}(\mathcal{X} ; \mathcal{Y})$; furthermore, we recursively define

$$
\mathcal{B}\left(\mathcal{X}_{1}, \ldots, \mathcal{X}_{r} ; \mathcal{Y}\right):=\mathcal{B}\left(\mathcal{X}_{1} ; \mathcal{B}\left(\mathcal{X}_{2}, \ldots, \mathcal{X}_{r} ; \mathcal{Y}\right)\right)
$$

and the special case

$$
\mathcal{B}^{0}(\mathcal{X} ; \mathcal{Y}):=\mathcal{Y} \quad \text { and } \quad \mathcal{B}^{r+1}(\mathcal{X} ; \mathcal{Y}):=\mathcal{B}\left(\mathcal{X} ; \mathcal{B}^{r}(\mathcal{X} ; \mathcal{Y})\right)
$$

For $\mathbf{T} \in \mathcal{B}\left(\mathcal{X}_{1}, \ldots, \mathcal{X}_{r} ; \mathcal{Y}\right)$ and $\mathbf{v}_{j} \in \mathcal{X}_{j}$ we use the shorthand notation $\mathbf{T v}_{1} \cdots \mathbf{v}_{r}:=$ $\mathbf{T}\left(\mathbf{v}_{1}, \ldots, \mathbf{v}_{r}\right) \in \mathcal{Y}$.

For a given Banach space $\mathcal{X}$ and a complete measure space $\mathcal{M}$ with measure $\mu$ the space $L_{\mu}^{p}(\mathcal{M} ; \mathcal{X})$ for $1 \leq p \leq \infty$ denotes the Bochner space, see [25], which contains all equivalence classes of strongly measurable functions $v: \mathcal{M} \rightarrow \mathcal{X}$ with finite norm

$$
\|v\|_{p, \mathcal{M} ; \mathcal{X}}:=\|v\|_{L_{\mu}^{p}(\mathcal{M} ; \mathcal{X})}:= \begin{cases}{\left[\int_{\mathcal{M}}\|v(x)\|_{\mathcal{X}}^{p} \mathrm{~d} \mu(x)\right]^{1 / p},} & p<\infty, \\ \underset{x \in \mathcal{M}}{\operatorname{ess~sup}\|v(x)\|_{\mathcal{X}},} & p=\infty .\end{cases}
$$

A function $v: \mathcal{M} \rightarrow \mathcal{X}$ is strongly measurable if there exists a sequence of countably-valued measurable functions $v_{n}: \mathcal{M} \rightarrow \mathcal{X}$, such that for almost every $m \in \mathcal{M}$ we have $\lim _{n \rightarrow \infty} v_{n}(m)=v(m)$. Note that, for finite measures $\mu$, we also have the usual inclusion $L_{\mu}^{p}(\mathcal{M} ; \mathcal{X}) \supset L_{\mu}^{q}(\mathcal{M} ; \mathcal{X})$ for $1 \leq p<q \leq \infty$.

For a given Banach space $\mathcal{X}$ and an open domain $\mathcal{D} \subset \mathbb{R}^{d}$, with $d \in \mathbb{N}^{*}$, the space $W^{\eta, p}(\mathcal{D} ; \mathcal{X})$ for $\eta \in \mathbb{N}$ and $1 \leq p \leq \infty$ denotes the Sobolev-Bochner space, which contains all equivalence classes of strongly measurable functions $v: \mathcal{D} \rightarrow \mathcal{X}$, such that the function itself and all weak derivatives up to total order $\eta$ are in $L^{p}(\mathcal{D} ; \mathcal{X})$ with the norm

$$
\|v\|_{\eta, p, \mathcal{D} ; \mathcal{X}}:=\|v\|_{W^{\eta, p}(\mathcal{D} ; \mathcal{X})}:=\sum_{|\boldsymbol{\alpha}| \leq \eta} \frac{1}{\boldsymbol{\alpha} !}\left\|\partial_{\mathbf{x}}^{\boldsymbol{\alpha}} v\right\|_{p, \mathcal{D} ; \mathcal{X}} .
$$

Moreover, $W_{0}^{\eta, p}(\mathcal{D} ; \mathcal{X})$ denotes the closure of the linear subspace of smooth functions with compact support, $C_{c}^{\infty}(\mathcal{D} ; \mathcal{X})$, in $W^{\eta, p}(\mathcal{D} ; \mathcal{X})$ and we set $H^{\eta}(\mathcal{D} ; \mathcal{X}):=$ $W^{\eta, 2}(\mathcal{D} ; \mathcal{X})$ and $H_{0}^{\eta}(\mathcal{D} ; \mathcal{X}):=W_{0}^{\eta, 2}(\mathcal{D} ; \mathcal{X})$. 
In the notation for the Bochner and Sobolev-Bochner spaces we may omit specifying the Banach space $\mathcal{X}$ when $\mathcal{X}=\mathbb{R}$. Especially, $H^{-\eta}(\mathcal{D})$ denotes the topological dual space of $H_{0}^{\eta}(\mathcal{D})$. Moreover, if the $\mathcal{X}$ we are considering is itself a Bochner or Sobolev-Bochner space then we replace the $\mathcal{X}$ in the subscript of the norm with the subscripts of its norm, for example

$$
\|v\|_{p, \mathcal{M} ; \eta, q, \mathcal{D} ; \mathcal{Y}}=\|v\|_{p, \mathcal{M} ; W^{\eta, q}(\mathcal{D} ; \mathcal{Y})}=\|v\|_{L_{\mu}^{p}\left(\mathcal{M} ; W^{\eta, q}(\mathcal{D} ; \mathcal{Y})\right)} .
$$

As we will need the Faà di Bruno formula, see [10], we just restate it here in a slightly adapted way, for our notation and usage - as a remark for reference.

Remark 1 Let $\mathcal{Y}$ and $\mathcal{Z}$ be Banach spaces, and $\mathcal{D} \subset \mathbb{R}^{m}$ or $\mathcal{D} \subset \mathbb{R}^{\mathbb{N}^{*}}$ a domain. Given $\mathbf{W}: Y \rightarrow \mathcal{Z}$ and $\mathbf{v}: \mathcal{D} \rightarrow \mathcal{Y}$, where $Y \subset \mathcal{Y}$ is open with $\operatorname{img}_{\mathcal{D}} \mathbf{v} \subset Y$ and $\mathbf{W}, \mathbf{v}$ are both sufficiently differentiable for the formula to make sense, then

$$
\partial_{\mathbf{x}}^{\boldsymbol{\alpha}}(\mathbf{W} \circ \mathbf{v})=\boldsymbol{\alpha} ! \sum_{r=1}^{|\boldsymbol{\alpha}|} \frac{1}{r !} \sum_{C(\boldsymbol{\alpha}, r)}\left(D^{r} \mathbf{W} \circ \mathbf{v}\right) \partial_{\mathbf{x}}^{\boldsymbol{\beta}_{1}} \mathbf{v} \cdots \partial_{\mathbf{x}}^{\boldsymbol{\beta}_{r}} \mathbf{v} \prod_{j=1}^{r} \frac{1}{\boldsymbol{\beta}_{j} !}
$$

for $\boldsymbol{\alpha} \in \mathbb{N}^{m}$, respectively $\boldsymbol{\alpha} \in \mathbb{N}_{f}^{\mathbb{N}^{*}}$ with $\boldsymbol{\alpha} \neq \mathbf{0}$, where $C(\boldsymbol{\alpha}, r)$ is the set of multiindex compositions of a multi-index $\boldsymbol{\alpha}$ into $r$ non-vanishing multi-indices, given by

$$
C(\boldsymbol{\alpha}, r):=\left\{\left(\boldsymbol{\beta}_{1}, \ldots, \boldsymbol{\beta}_{r}\right) \in\left(\mathbb{N}_{f}^{\mathbb{N}^{*}}\right)^{r}: \sum_{j=1}^{r} \boldsymbol{\beta}_{j}=\boldsymbol{\alpha} \text { and } \boldsymbol{\beta}_{j} \neq \mathbf{0} \text { for all } 1 \leq j \leq r\right\}
$$

Finally, we note that to avoid the use of generic but unspecified constants in certain formulas we use $c \lesssim d$ to mean that $c$ can be bounded by a multiple of $d$, independently of parameters which $c$ and $d$ may depend on. Obviously, $c \gtrsim d$ is defined as $d \lesssim c$ and we write $c \approx d$ if $c \lesssim d$ and $c \gtrsim d$.

\subsection{Model problem}

Let $(\Omega, \mathcal{F}, \mathbb{P})$ be a separable, complete probability space. Then, we consider the following second order diffusion problem with a random anisotropic diffusion coefficient

$$
\text { for almost every } \omega \in \Omega:\left\{\begin{aligned}
-\operatorname{div}_{\mathbf{x}}\left(\mathbf{A}[\omega] \nabla_{\mathbf{x}} u[\omega]\right)=f & \text { in } D, \\
u[\omega]=0 & \text { on } \partial D,
\end{aligned}\right.
$$

where $D \subset \mathbb{R}^{d}$ is a Lipschitz domain with $d \geq 1$, the function $f \in H^{-1}(D)$ describes the known source and the random anisotropic diffusion coefficient, $\mathbf{A} \in$ $L_{\mathbb{P}}^{\infty}\left(\Omega ; L^{\infty}\left(D ; \mathbb{R}_{\mathrm{symm}}^{d \times d}\right)\right)$, is given by

$$
\mathbf{A}[\omega](\mathbf{x})=\mathbf{D}(\mathbf{V}[\omega](\mathbf{x})) .
$$

Here, $\mathbf{V} \in L_{\mathbb{P}}^{\infty}\left(\Omega ; L^{\infty}\left(D ; \mathbb{R}^{d}\right)\right)$ is a random vector-valued field that specifies the direction and diffusion strength of a notable direction at each point and $\mathbf{D}: \mathbb{R}^{d} \rightarrow$ $\mathbb{R}_{\text {symm }}^{d \times d}$ is a mapping, which is given by

$$
\mathbf{D}(\mathbf{v}):=a \mathbf{I}+\left(\|\mathbf{v}\|_{2}-a\right) \frac{\mathbf{v v}^{\top}}{\mathbf{v}^{\top} \mathbf{v}}
$$


for $a \in \mathbb{R}$ a given positive number and which maps this description of direction and diffusion strength given by $\mathbf{v} \in \mathbb{R}^{d}$ to a diffusion coefficient matrix that accounts for homogeneous diffusion with strength $a$ perpendicular to $\mathbf{v}$ and diffusion of strength $\|\mathbf{v}\|_{2}$ in the direction of $\mathbf{v}$.

We require that there exist $\underline{a}>0$ and $\bar{a}>0$ such that

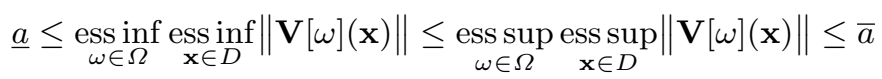

and $\underline{a} \leq a \leq \bar{a}$. Then, by definition, A satisfies the uniform ellipticity condition

$$
\underline{a} \leq \underset{\omega \in \Omega}{\operatorname{ess} \inf } \underset{\mathbf{x} \in D}{\operatorname{essinf}} \lambda_{\min }(\mathbf{A}[\omega](\mathbf{x})) \leq \underset{\omega \in \Omega}{\operatorname{ess} \sup } \underset{\mathbf{x} \in D}{\operatorname{ess} \sup } \lambda_{\max }(\mathbf{A}[\omega](\mathbf{x})) \leq \bar{a},
$$

where $\lambda_{\min }(\mathbf{B})$ and $\lambda_{\max }(\mathbf{B})$ denote the smallest and largest eigenvalues of the

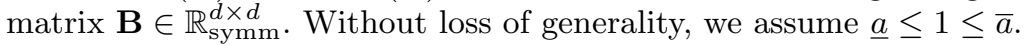

It is assumed that the spatial variable $\mathbf{x}$ and the stochastic parameter $\omega$ of the random field have been separated by the Karhunen-Loève expansion of $\mathbf{V}$, yielding a parametrised expansion

$$
\mathbf{V}[\mathbf{y}](\mathbf{x})=\mathbb{E}[\mathbf{V}](\mathbf{x})+\sum_{k=1}^{\infty} \sigma_{k} \boldsymbol{\psi}_{k}(\mathbf{x}) y_{k}
$$

where $\mathbf{y}=\left(y_{k}\right)_{k \in \mathbb{N}^{*}} \in \square:=\mathbb{R}^{\mathbb{N}^{*}}$ is a sequence of uncorrelated random variables, see e.g. [24]. In the following, we will denote the completion of the pushforward of the measure $\mathbb{P}$ on $\square$ equipped with its product $\sigma$-Algebra of the Borel $\sigma$-Algebras of $\mathbb{R}$ as $\mathbb{P}_{\mathbf{y}}$. Then, we also view $\mathbf{A}[\mathbf{y}](\mathbf{x})$ and $u[\mathbf{y}](\mathbf{x})$ as being parametrised by $\mathbf{y}$ and restate (1) as

$$
\text { for almost every } \mathbf{y} \in \square:\left\{\begin{aligned}
-\operatorname{div}_{\mathbf{x}}\left(\mathbf{A}[\mathbf{y}] \nabla_{\mathbf{x}} u[\mathbf{y}]\right)=f & \text { in } D, \\
u[\mathbf{y}]=0 & \text { on } \partial D .
\end{aligned}\right.
$$

Lastly, we note that the spatially weak form of (6) is then given by

$$
\left\{\begin{array}{l}
\text { Find } u \in L_{\mathbb{P}_{\mathbf{y}}}^{\infty}\left(\square ; H_{0}^{1}(D)\right) \text { such that } \\
\quad\left(\mathbf{A}[\mathbf{y}] \nabla_{\mathbf{x}} u[\mathbf{y}], \nabla_{\mathbf{x}} v\right)_{L^{2}\left(D ; \mathbb{R}^{d}\right)}=(f, v)_{L^{2}\left(D ; \mathbb{R}^{d}\right)} \\
\text { for almost every } \mathbf{y} \in \square \text { and all } v \in H_{0}^{1}(D) .
\end{array}\right.
$$

This also entails the well known stability estimate.

Lemma 1 There is a unique solution $u \in L_{\mathbb{P}_{\mathbf{y}}}^{\infty}\left(\square ; H_{0}^{1}(D)\right)$ of $(7)$, which fulfils

$$
\|u\|_{\infty, \square ; 1,2, D} \leq \frac{1}{\underline{a} c_{V}^{2}}\|f\|_{-1,2, D},
$$

where $c_{V}$ is the Poincaré-Friedrichs constant of $H_{0}^{1}(D)$. 


\section{Parametric regularity and multilevel quadrature}

We now derive regularity estimates for the solution $u$ of (7) and apply multilevel quadrature for approximating the mean of $u$. The regularity estimates are based on the following assumption on the decay of the expansion of $\mathbf{V}$.

Assumption 1 We assume that $\mathbb{E}[\mathbf{V}]$ and the $\boldsymbol{\psi}_{k}$ are elements of $W^{\tau, \infty}\left(D ; \mathbb{R}^{d}\right)$ for a $\tau \in \mathbb{N}$ and that the sequence $\gamma_{\tau}=\left(\gamma_{\tau, k}\right)_{k \in \mathbb{N}^{*}}$, given by

$$
\gamma_{\tau, k}:=\left\|\sigma_{k} \psi_{k}\right\|_{\tau, \infty, D ; \mathbb{R}^{d}},
$$

is at least in $L^{1}\left(\mathbb{N}^{*} ; \mathbb{R}\right)$. We also define

$$
c_{\boldsymbol{\gamma}_{\tau}}:=\max \left\{\|\mathbb{E}[\mathbf{V}]\|_{\tau, \infty, D ; \mathbb{R}^{d}}+\left\|\boldsymbol{\gamma}_{\tau}\right\|_{1, \mathbb{N}^{*} ; \mathbb{R}}, 1\right\} .
$$

Furthermore, for the regularity estimates we also require an elliptic regularity result.

Assumption 2 Let $\mathcal{R}_{\tau}$ be a Banach space with norm $\|\cdot\|_{\mathcal{R}_{\tau}}$ such that, for all

$$
\mathbf{B} \in W^{\tau, \infty}\left(D ; \mathbb{R}_{\mathrm{symm}}^{d \times d}\right) \cap \mathcal{R}_{\tau}
$$

that fulfil (4), we have that the problem of solving

$$
\left(\mathbf{B} \nabla_{\mathbf{x}} u, \nabla_{x} v\right)_{L^{2}\left(D ; \mathbb{R}^{d}\right)}=(h, v)_{L^{2}(D)}
$$

for any $h \in H^{\tau-1}(D)$ has a unique solution $u \in H_{0}^{1}(D)$, which also lies in $H^{\tau+1}(D)$, with

$$
\|u\|_{\tau+1,2, D} \leq C_{\tau, e r}\left(D, \underline{a},\|\mathbf{B}\|_{\mathcal{R}_{\tau}}\right)\|h\|_{\tau-1,2, D},
$$

where $C_{\tau, \text { er }}$ only depends on $D, \underline{\text { a }}$ and continuously on $\|\mathbf{B}\|_{\mathcal{R}_{\tau}}$.

We assume from here on that $\mathbf{A}$ also lies in $L_{\mathbb{P}_{\mathbf{y}}}^{\infty}\left(\square ; \mathcal{R}_{\tau}\right)$.

Note, that for $\tau=0$, this reduces to the stability estimate, for which the parametric regularity may be found in [24]. Therefore, we will hereafter only consider the case where $\tau \geq 1$. Such an elliptic regularity estimate for example is known for $\tau=1$, when the domain is convex and bounded and $\mathcal{R}_{\tau}=C^{0,1}\left(\bar{D} ; \mathbb{R}^{d \times d}\right)$, see [16, Theorems 3.2.1.2 and 3.1.3.1]. The elliptic regularity estimate is also known to hold for $\tau \geq 1$ and $d=2$, when the domain's boundary is smooth and $\mathcal{R}_{\tau}=$ $W^{\tau, \infty}\left(D ; \mathbb{R}^{d \times \bar{d}}\right)$, see [7].

The rest of this section is now split into three subsections. The first subsection is dedicated to the computation of explicit bounds for the regularity of the mapping $\mathbf{D}$, which maps our description of a fibre to a diffusion coefficient. With this result at hand we then, in the second subsection, may assert regularity estimates for the diffusion coefficient itself and derive the regularity estimates of the solution $u$ in Theorem 3. We then briefly discuss what kind of convergence rates and computational complexity this regularity of $u$ yields, for the example of approximating $\mathbb{E}[u]$ using singlelevel and multilevel quadrature methods, in the third subsection. 
3.1 Regularity of the vector field to diffusion coefficient mapping

Since our aim here is to give explicit bounds for the regularity of the mapping $\mathbf{D}$, which maps our description of a fibre to a diffusion coefficient. We first supply ${ }^{1}$ the following three lemmas that give simple explicit upper bounds on the constants which bound the derivatives of a real analytic function that is defined by summing, multiplying or composing real analytic functions between Banach spaces.

Lemma 2 Let $\mathcal{X}$ and $\mathcal{Y}$ be Banach spaces, $X \subset \mathcal{X}$ and $\mathbf{v}_{1}, \ldots, \mathbf{v}_{t}: X \rightarrow \mathcal{Y}$ be infinitely Fréchet differentiable mappings such that there exist some constants $k_{i}, c_{i} \geq 0$ with

$$
\left\|\mathrm{D}^{r} \mathbf{v}_{i}(\mathbf{x})\right\|_{\mathcal{B}^{r}(\mathcal{X} ; \mathcal{Y})} \leq r ! k_{i} c_{i}^{r}
$$

for all $\mathbf{x} \in X, i=1, \ldots, t$. Then, the mapping $\mathbf{v}_{1}+\cdots+\mathbf{v}_{t}$ is infinitely Fréchet differentiable with

$$
\left\|\mathrm{D}^{r}\left(\mathbf{v}_{1}+\cdots+\mathbf{v}_{t}\right)(\mathbf{x})\right\|_{\mathcal{B}^{r}(\mathcal{X} ; \mathcal{Y})} \leq r ! k c^{r}
$$

for all $\mathbf{x} \in X$, with $k:=k_{1}+\cdots+k_{t}$ and $c:=\max \left\{c_{1}, \ldots, c_{t}\right\}$.

Lemma 3 Let $\mathcal{X}, \mathcal{Y}_{1}, \ldots, \mathcal{Y}_{t}$, and $\mathcal{Z}$ be Banach spaces, $X \subset \mathcal{X}$ and $\mathbf{M}: X \rightarrow$ $\mathcal{B}\left(\mathcal{Y}_{1}, \ldots, \mathcal{Y}_{t} ; \mathcal{Z}\right), \mathbf{v}_{i}: X \rightarrow \mathcal{Y}_{i}$ be infinitely Fréchet differentiable mappings such that there exist some constants $k_{\mathbf{M}}, c_{\mathbf{M}}, k_{i}, c_{i} \geq 0$ with

$$
\left\|\mathrm{D}^{r} \mathbf{M}(\mathbf{x})\right\|_{\mathcal{B}^{r}\left(\mathcal{X} ; \mathcal{B}\left(\mathcal{Y}_{1}, \ldots, \mathcal{Y}_{t} ; \mathcal{Z}\right)\right)} \leq r ! k_{\mathbf{M}} c_{\mathbf{M}}^{r}
$$

and

$$
\left\|\mathrm{D}^{r} \mathbf{v}_{i}(\mathbf{x})\right\|_{\mathcal{B}^{r}\left(\mathcal{X} ; \mathcal{Y}_{i}\right)} \leq r ! k_{i} c_{i}^{r}
$$

for all $\mathbf{x} \in X, i=1, \ldots, t$. Then, the mapping $\mathbf{M} \mathbf{v}_{1} \cdots \mathbf{v}_{t}$ is infinitely Fréchet differentiable with

$$
\left\|\mathrm{D}^{r}\left(\mathbf{M v}_{1} \cdots \mathbf{v}_{t}\right)(\mathbf{x})\right\|_{\mathcal{B}^{r}(\mathcal{X} ; \mathcal{Z})} \leq r ! k c^{r}
$$

for all $\mathbf{x} \in X$, with $k:=k_{\mathbf{M}} k_{1} \cdots k_{t}$ and $c:=2^{t} \max \left\{c_{\mathbf{M}}, c_{1}, \ldots, c_{t}\right\}$.

Lemma 4 Let $\mathcal{X}, \mathcal{Y}$, and $\mathcal{Z}$ be Banach spaces, $X \subset \mathcal{X}, Y \subset \mathcal{Y}$ and $\mathbf{W}: Y \rightarrow \mathcal{Z}$, $\mathbf{v}: X \rightarrow \mathcal{Y}$ both be infinitely Fréchet differentiable mappings such that there exist some constants $k_{\mathbf{W}}, c_{\mathbf{W}}, k_{\mathbf{v}}, c_{\mathbf{v}} \geq 0$ with

$$
\left\|\mathrm{D}^{r} \mathbf{W}(\mathbf{y})\right\|_{\mathcal{B}^{r}(\mathcal{Y} ; \mathcal{Z})} \leq r ! k_{\mathbf{W}} c_{\mathbf{W}}^{r}
$$

and

$$
\left\|\mathrm{D}^{r} \mathbf{v}(\mathbf{x})\right\|_{\mathcal{B}^{r}(\mathcal{X} ; \mathcal{Y})} \leq r ! k_{\mathbf{v}} c_{\mathbf{v}}^{r}
$$

for all $\mathbf{x} \in X$ and $\mathbf{y} \in Y$. Furthermore, let $\mathbf{v}(X) \subset Y$, then, the mapping $\mathbf{W} \circ \mathbf{v}$ is infinitely Fréchet differentiable with

$$
\left\|\mathrm{D}^{r}(\mathbf{W} \circ \mathbf{v})(\mathbf{x})\right\|_{\mathcal{B}^{r}(\mathcal{X} ; \mathcal{Z})} \leq r ! k c^{r}
$$

for all $\mathbf{x} \in X$, with $k:=k_{\mathbf{W}}$ and $c:=\left(c_{\mathbf{W}} k_{\mathbf{v}}+1\right) c_{\mathbf{v}}$.

1 We omit their proofs, as the first lemmma essentially follows from the linearity of the Fréchet derivative, the second can be proved by an iterated use of the Leibniz formula for Fréchet derivatives and the third one is a simple modification of the proof shown in [27, proof of Proposition 1.4.2] for the composition of real analytic functions from $\mathbb{R}$ to $\mathbb{R}$. 
By using these results we can now consider our specific mapping $\mathbf{D}$, see (2)

$$
\mathbf{D}(\mathbf{v}):=a \mathbf{I}+\left(\|\mathbf{v}\|_{2}-a\right) \frac{\mathbf{v} \mathbf{v}^{\top}}{\mathbf{v}^{\top} \mathbf{v}}
$$

While we restrict ourselves to this mapping, the above results clearly can be used to also consider other mappings; e.g. the other diffusion coefficent stated in the introduction may also be stated in the form $\mathbf{A}=\mathbf{D} \circ \mathbf{V}$, where $\mathbf{V}$ is the concatenation of $\mathbf{f}, \mathbf{t}, \mathbf{s}$ and $\left[\alpha_{1}, \alpha_{2}, \alpha_{3}\right]$.

Theorem 1 Let $V:=\left\{\mathbf{v} \in \mathbb{R}^{d}: \underline{a} \leq\|\mathbf{v}\|_{2} \leq \bar{a}\right\}$ then the mapping $\mathbf{D}$ defined in (2) is infinitely Fréchet differentiable on $V$ with

$$
\left\|\mathrm{D}^{r} \mathbf{D}(\mathbf{v})\right\|_{\mathcal{B}^{r}\left(\mathbb{R}^{d} ; \mathbb{R}_{\mathrm{symm}}^{d \times d}\right)} \leq r ! k_{\mathbf{D}} c_{\mathbf{D}}^{r}
$$

for all $\mathbf{v} \in V$, with $k_{\mathbf{D}}:=\bar{a}+2 \bar{a}^{3} \underline{a}^{-2}$ and $c_{\mathbf{D}}:=\left(\bar{a}^{2} \underline{a}^{-2}+1\right) 8$.

Proof One starts from the mappings

$$
\mathbf{D}_{1}: V \rightarrow \mathbb{R}^{d}, \mathbf{v} \mapsto \mathbf{v} \quad \text { and } \quad \mathbf{D}_{2}: V \rightarrow \mathbb{R}^{1 \times d}, \mathbf{v} \mapsto \mathbf{v}^{\top}
$$

which are obviously infinitely Fréchet differentiable with the bounds

$$
\left\|\mathrm{D}^{r} \mathbf{D}_{1}(\mathbf{v})\right\|_{\mathcal{B}^{r}\left(\mathbb{R}^{d} ; \mathbb{R}^{d}\right)} \leq r ! k_{1} c_{1}^{r} \quad \text { and } \quad\left\|\mathrm{D}^{r} \mathbf{D}_{2}(\mathbf{v})\right\|_{\mathcal{B}^{r}\left(\mathbb{R}^{d} ; \mathbb{R}^{1 \times d}\right)} \leq r ! k_{2} c_{2}^{r}
$$

for all $\mathbf{v} \in V$, with $k_{1}=k_{2}=\bar{a}$ and $c_{1}=c_{2}=1$. Then, one may stepwise build up the mapping $\mathbf{D}$ by applying Lemmas 2 and 3 as well as Lemma 4 with the outer functions $v(x)=x^{-1}$ and $w(x)=\sqrt{x}$.

Especially, we remark that the $r$-th derivative of $v$ and $w$ are given by

$$
\mathrm{D}_{x}^{r} v(x) h_{1} \cdots h_{r}=(-1)^{r} r ! x^{-1-r} \prod_{k=1}^{r} h_{k}=(-1)^{r} r ! v(x) v(x)^{r} \prod_{k=1}^{r} h_{k}
$$

and

$$
\mathrm{D}_{x}^{r} w(x) h_{1} \cdots h_{r}=c_{r} x^{\frac{1}{2}-r} \prod_{k=1}^{r} h_{k}=c_{r} w(x) v(x)^{r} \prod_{k=1}^{r} h_{k}
$$

where $c_{r}:=\prod_{i=0}^{r-1}\left(\frac{1}{2}-r\right)$. For $x \in\left[\underline{a}^{2}, \bar{a}^{2}\right]$ we therefore have

$$
\left\|\mathrm{D}_{x}^{r} v(x)\right\|_{\mathcal{B}^{r}(\mathbb{R} ; \mathbb{R})} \leq r ! k_{v} c_{v}^{r} \quad \text { and } \quad\left\|\mathrm{D}_{x}^{r} w(x)\right\|_{\mathcal{B}^{r}(\mathbb{R} ; \mathbb{R})} \leq r ! k_{w} c_{w}^{r}
$$

where $k_{v}=\underline{a}^{-2}$ and $c_{v}=\underline{a}^{-2}$ as well as $k_{w}=\bar{a}$ and $c_{w}=\underline{a}^{-2}$.

Thus, the assertion follows - with some book keeping necessary for the constants. 
3.2 Parametric regularity

First, we provide some results concerning the norms introduced beforehand that are subsequently used in this subsection. This mainly stems from the situation that in general we will be considering elements of spaces of the form $L_{\mathbb{P}_{\mathbf{y}}}^{\infty}(\square ; \mathcal{X})$; indeed, we therefore also introduce the shorthand notation

$$
\|v\|\left\|_{\mathcal{X}}:=\right\| v \|_{\infty, \square ; \mathcal{X}} .
$$

We will especially make use it for spaces of the form $L_{\mathbb{P}_{\mathbf{y}}}^{\infty}\left(\square ; W^{\eta, p}(D ; \mathcal{X})\right)$, where this then becomes $\|v\|_{\eta, p, D ; \mathcal{X}}=\|v\|_{\infty, \square ; \eta, p, D ; \mathcal{X}}$. With these lemmas at hand, we then first prove the parametric regularity of the diffusion coefficient $\mathbf{A}$ and then also the parametric regularity of the solution $u$ of (7).

The following lemma gives a bound after applying a $\operatorname{div}_{\mathbf{x}}$ or $\nabla_{\mathbf{x}}$.

Lemma 5 Let $\eta \in \mathbb{N}^{*}, 1 \leq p \leq \infty$. For $\mathbf{v} \in L_{\mathbb{P}_{\mathbf{y}}}^{\infty}\left(\square ; W^{\eta, p}\left(D ; \mathbb{R}^{d}\right)\right)$ we have that $\operatorname{div}_{\mathbf{x}} \mathbf{v} \in L_{\mathbb{P}_{\mathbf{y}}}^{\infty}\left(\square ; W^{\eta-1, p}(D ; \mathbb{R})\right)$ with

$$
\left\|\operatorname{div}_{\mathbf{x}} \mathbf{v}\right\|_{\eta-1, p, D} \leq \eta d\|\mathbf{v}\|_{\eta, p, D ; \mathbb{R}^{d}} .
$$

For $v \in L_{\mathbb{P}_{\mathbf{y}}}^{\infty}\left(\square ; W^{\eta, p}(D)\right)$ we have that $\nabla_{\mathbf{x}} v \in L_{\mathbb{P}_{\mathbf{y}}}^{\infty}\left(\square ; W^{\eta-1, p}\left(D ; \mathbb{R}^{d}\right)\right)$ with

$$
\left\|\nabla_{\mathbf{x}} v\right\|_{\eta-1, p, D ; \mathbb{R}^{d}} \leq \eta d\|v\|_{\eta, p, D} .
$$

Proof We calculate

$$
\begin{gathered}
\left\|\operatorname{div}_{\mathbf{x}} \mathbf{v}[\mathbf{y}]\right\|_{\eta-1, p, D}=\left\|\sum_{i=1}^{d} \partial_{x_{i}} v_{i}[\mathbf{y}]\right\|_{\eta-1, p, D}=\sum_{|\boldsymbol{\alpha}| \leq \eta-1} \frac{1}{\boldsymbol{\alpha} !}\left\|\sum_{i=1}^{d} \partial_{\mathbf{x}}^{\boldsymbol{\alpha}} \partial_{x_{i}} v_{i}[\mathbf{y}]\right\|_{p, D} \\
\leq \sum_{|\boldsymbol{\alpha}| \leq \eta-1} \frac{1}{\boldsymbol{\alpha} !} \sum_{i=1}^{d}\left\|\partial_{\mathbf{x}}^{\boldsymbol{\alpha}} \partial_{x_{i}} v_{i}[\mathbf{y}]\right\|_{p, D} \leq \eta \sum_{|\boldsymbol{\alpha}| \leq \eta} \frac{1}{\boldsymbol{\alpha} !} \sum_{i=1}^{d}\left\|\partial_{\mathbf{x}}^{\boldsymbol{\alpha}} v_{i}[\mathbf{y}]\right\|_{p, D} \\
\leq \eta d \sum_{|\boldsymbol{\alpha}| \leq \eta} \frac{1}{\boldsymbol{\alpha} !}\left\|\partial_{\mathbf{x}}^{\boldsymbol{\alpha}} \mathbf{v}[\mathbf{y}]\right\|_{p, D ; \mathbb{R}^{d}} \leq \eta d\|\mathbf{v}[\mathbf{y}]\|_{\eta, p, D ; \mathbb{R}^{d}} .
\end{gathered}
$$

We also may compute

$$
\begin{aligned}
& \left\|\nabla_{\mathbf{x}} v[\mathbf{y}]\right\|_{\eta-1, p, D ; \mathbb{R}^{d}}=\sum_{|\boldsymbol{\alpha}| \leq \eta-1} \frac{1}{\boldsymbol{\alpha} !}\left\|\partial_{\mathbf{x}}^{\boldsymbol{\alpha}} \nabla_{\mathbf{x}} v[\mathbf{y}]\right\|_{p, D ; \mathbb{R}^{d}} \\
& \quad=\sum_{|\boldsymbol{\alpha}| \leq \eta-1} \frac{1}{\boldsymbol{\alpha} !}\left\|\left[\begin{array}{c}
\partial_{\mathbf{x}}^{\boldsymbol{\alpha}} \partial_{x_{1}} v[\mathbf{y}] \\
\vdots \\
\partial_{\mathbf{x}}^{\boldsymbol{\alpha}} \partial_{x_{d}} v[\mathbf{y}]
\end{array}\right]\right\|_{p, D ; \mathbb{R}^{d}} \leq \sum_{|\boldsymbol{\alpha}| \leq \eta-1} \frac{1}{\boldsymbol{\alpha} !} \sum_{i=1}^{d}\left\|\partial_{\mathbf{x}}^{\boldsymbol{\alpha}} \partial_{x_{i}} v[\mathbf{y}]\right\|_{p, D} \\
& \quad \leq \eta \sum_{|\boldsymbol{\alpha}| \leq \eta} \frac{1}{\boldsymbol{\alpha} !} \sum_{i=1}^{d}\left\|\partial_{\mathbf{x}}^{\boldsymbol{\alpha}} v[\mathbf{y}]\right\|_{p, D} \leq \eta d \sum_{|\boldsymbol{\alpha}| \leq \eta} \frac{1}{\boldsymbol{\alpha} !}\left\|\partial_{\mathbf{x}}^{\boldsymbol{\alpha}} v[\mathbf{y}]\right\|_{p, D} \leq \eta d\|v[\mathbf{y}]\|_{\eta, p, D} .
\end{aligned}
$$

Since these calculations hold for almost every $\mathbf{y} \in \square$, applying the essential supremum over $\mathbf{y} \in \square$ then yields the assertions.

The Leibniz rule also yields the following kind of submultiplicativity. 
Lemma 6 Let $\eta \in \mathbb{N}, 1 \leq p, p_{1}, \ldots, p_{r} \leq \infty, \mathcal{X}_{1}, \ldots, \mathcal{X}_{r}$ and $\mathcal{Y}$ be Banach spaces and

$$
\mathbf{M} \in W^{\eta, p}\left(D ; \mathcal{B}\left(\mathcal{X}_{1}, \ldots, \mathcal{X}_{r} ; \mathcal{Y}\right)\right), \quad \mathbf{v}_{j} \in W^{\eta, p_{j}}\left(D ; \mathcal{X}_{j}\right)
$$

with $q=\left(p^{-1}+p_{1}^{-1}+\cdots+p_{r}^{-1}\right)^{-1} \geq 1$. Then, we have

$$
\left\|\mathbf{M} \mathbf{v}_{1} \cdots \mathbf{v}_{r}\right\|_{\eta, q, D ; \mathcal{Y}} \leq\|\mathbf{M}\|_{\eta, p, D ; \mathcal{B}\left(\mathcal{X}_{1}, \ldots, \mathcal{X}_{r} ; \mathcal{Y}\right)} \prod_{j=1}^{r}\left\|\mathbf{v}_{j}\right\|_{\eta, p_{j}, D ; \mathcal{X}_{j}} .
$$

Proof If the assertion holds for $r=1$ then by induction it also follows for $r>1$. Therefore, we only consider the case with $r=1$ and set $\mathbf{v}:=\mathbf{v}_{1}$ and $p^{\prime}=p_{1}$.

Let $\boldsymbol{\alpha}, \boldsymbol{\beta} \in \mathbb{N}^{d}$ be two multi-indices, then we have

$$
\frac{1}{(\boldsymbol{\alpha}+\boldsymbol{\beta}) !}\left(\begin{array}{c}
\boldsymbol{\alpha}+\boldsymbol{\beta} \\
\boldsymbol{\beta}
\end{array}\right)=\frac{1}{(\boldsymbol{\alpha}+\boldsymbol{\beta}) !} \frac{(\boldsymbol{\alpha}+\boldsymbol{\beta}) !}{\boldsymbol{\alpha} ! \boldsymbol{\beta} !}=\frac{1}{\boldsymbol{\alpha} ! \boldsymbol{\beta} !} .
$$

We now can calculate

$$
\begin{aligned}
\|\mathbf{M v}\|_{\eta, q, D ; \mathcal{Y}} & =\sum_{|\boldsymbol{\alpha}| \leq \eta} \frac{1}{\boldsymbol{\alpha} !}\left\|\partial_{\mathbf{x}}^{\boldsymbol{\alpha}}[\mathbf{M v}]\right\|_{q, D ; \mathcal{Y}} \\
& =\sum_{|\boldsymbol{\alpha}| \leq \eta} \frac{1}{\boldsymbol{\alpha} !}\left\|\sum_{\boldsymbol{\beta} \leq \boldsymbol{\alpha}}\left(\begin{array}{l}
\boldsymbol{\alpha} \\
\boldsymbol{\beta}
\end{array}\right) \partial_{\mathbf{x}}^{\boldsymbol{\beta}} \mathbf{M} \partial_{\mathbf{x}}^{\boldsymbol{\alpha}-\boldsymbol{\beta}} \mathbf{v}\right\|_{q, D ; \mathcal{Y}} \\
& \leq \sum_{|\boldsymbol{\alpha}| \leq \eta} \sum_{\boldsymbol{\beta} \leq \boldsymbol{\alpha}} \frac{1}{\boldsymbol{\alpha} !}\left(\begin{array}{l}
\boldsymbol{\alpha} \\
\boldsymbol{\beta}
\end{array}\right)\left\|\partial_{\mathbf{x}}^{\boldsymbol{\beta}} \mathbf{M} \partial_{\mathbf{x}}^{\boldsymbol{\alpha}-\boldsymbol{\beta}} \mathbf{v}\right\|_{q, D ; \mathcal{Y}} \\
& \leq \sum_{|\boldsymbol{\alpha}| \leq \eta} \sum_{\boldsymbol{\beta} \leq \boldsymbol{\alpha}} \frac{1}{\boldsymbol{\alpha} !}\left(\begin{array}{l}
\boldsymbol{\alpha} \\
\boldsymbol{\beta}
\end{array}\right)\left\|\partial_{\mathbf{x}}^{\boldsymbol{\beta}} \mathbf{M}\right\|_{p, D ; \mathcal{B}(\mathcal{X} ; \mathcal{Y}))} \| \partial_{\mathbf{x}}^{\boldsymbol{\alpha}-\boldsymbol{\beta} \mathbf{v} \|_{p^{\prime}, D ; \mathcal{X}}}
\end{aligned}
$$

By a change of variables, i.e. replacing $\boldsymbol{\alpha}$ with $\boldsymbol{\alpha}+\boldsymbol{\beta}$, and remarking that

$$
\{(\boldsymbol{\alpha}-\boldsymbol{\beta}, \boldsymbol{\beta}):|\boldsymbol{\alpha}| \leq \eta, \boldsymbol{\beta} \leq \boldsymbol{\alpha}\}=\{(\boldsymbol{\alpha}, \boldsymbol{\beta}):|\boldsymbol{\alpha}|+|\boldsymbol{\beta}| \leq \eta\},
$$

we find the identity

$$
\begin{aligned}
& \sum_{|\boldsymbol{\alpha}| \leq \eta} \sum_{\boldsymbol{\beta} \leq \boldsymbol{\alpha}} \frac{1}{\boldsymbol{\alpha} !}\left(\begin{array}{c}
\boldsymbol{\alpha} \\
\boldsymbol{\beta}
\end{array}\right)\left\|\partial_{\mathbf{x}}^{\boldsymbol{\beta}} \mathbf{M}\right\|_{p, D ; \mathcal{B}(\mathcal{X} ; \mathcal{Y})}\left\|\partial_{\mathbf{x}}^{\boldsymbol{\alpha}-\boldsymbol{\beta}} \mathbf{v}\right\|_{p^{\prime}, D ; \mathcal{X}} \\
& =\sum_{|\boldsymbol{\alpha}|+|\boldsymbol{\beta}| \leq \eta} \frac{1}{(\boldsymbol{\alpha}+\boldsymbol{\beta}) !}\left(\begin{array}{c}
\boldsymbol{\alpha}+\boldsymbol{\beta} \\
\boldsymbol{\beta}
\end{array}\right)\left\|\partial_{\mathbf{x}}^{\boldsymbol{\beta}} \mathbf{M}\right\|_{p, D ; \mathcal{B}(\mathcal{X} ; \mathcal{Y})}\left\|\partial_{\mathbf{x}}^{\boldsymbol{\alpha}} \mathbf{v}\right\|_{p^{\prime}, D ; \mathcal{X}} \\
& =\sum_{|\boldsymbol{\alpha}|+|\boldsymbol{\beta}| \leq \eta} \frac{1}{\boldsymbol{\alpha} ! \boldsymbol{\beta} !}\left\|\partial_{\mathbf{x}}^{\boldsymbol{\beta}} \mathbf{M}\right\|_{p, D ; \mathcal{B}(\mathcal{X} ; \mathcal{Y})}\left\|\partial_{\mathbf{x}}^{\boldsymbol{\alpha}} \mathbf{v}\right\|_{p^{\prime}, D ; \mathcal{X}} .
\end{aligned}
$$

Consequently, as

$$
\begin{aligned}
\|\mathbf{M} \mathbf{v}\|_{\eta, q, D ; \mathcal{Y}} & \leq \sum_{|\boldsymbol{\alpha}|,|\boldsymbol{\beta}| \leq \eta} \frac{1}{\boldsymbol{\alpha} ! \boldsymbol{\beta} !}\left\|\partial_{\mathbf{x}}^{\boldsymbol{\beta}} \mathbf{M}\right\|_{p, D ; \mathcal{B}(\mathcal{X} ; \mathcal{Y})}\left\|\partial_{\mathbf{x}}^{\boldsymbol{\alpha}} \mathbf{v}\right\|_{p^{\prime}, D ; \mathcal{X}} \\
& \leq \sum_{|\boldsymbol{\beta}| \leq \eta} \frac{1}{\boldsymbol{\beta} !}\left\|\partial_{\mathbf{x}}^{\boldsymbol{\beta}} \mathbf{M}\right\|_{p, D ; \mathcal{B}(\mathcal{X} ; \mathcal{Y})} \sum_{|\boldsymbol{\alpha}| \leq \eta} \frac{1}{\boldsymbol{\alpha} !}\left\|\partial_{\mathbf{x}}^{\boldsymbol{\alpha}} \mathbf{v}\right\|_{p^{\prime}, D ; \mathcal{X}} \\
& =\|\mathbf{M}\|_{\eta, p, D ; \mathcal{B}(\mathcal{X} ; \mathcal{Y})}\|\mathbf{v}\|_{\eta, p^{\prime}, D ; \mathcal{X}}
\end{aligned}
$$

we arrive at the desired estimate. 
Lastly, the Faà di Bruno formula, see Remark 1, now yields the following lemma.

Lemma 7 Let $\eta \in \mathbb{N}, 1 \leq p \leq \infty, \mathcal{X}$ and $\mathcal{Y}$ be Banach spaces,

$$
\mathbf{v} \in W^{\eta, \infty}(D ; \mathcal{X})
$$

$X \subset \mathcal{X}$ be open with $\operatorname{img}_{D} \mathbf{v} \subset X, \mathbf{W}: X \rightarrow \mathcal{Y} \eta$-times Fréchet differentiable and, for $0 \leq r \leq \eta$,

$$
\mathrm{D}^{r} \mathbf{W} \circ \mathbf{v} \in L^{p}\left(D ; \mathcal{B}^{r}(\mathcal{X} ; \mathcal{Y})\right)
$$

Then, we have

$$
\mathbf{W} \circ \mathbf{v} \in W^{\eta, p}(D ; \mathcal{Y})
$$

with

$$
\|\mathbf{W} \circ \mathbf{v}\|_{\eta, p, D ; \mathcal{Y}} \leq \sum_{r=0}^{\eta} \frac{1}{r !}\left\|\mathrm{D}^{r} \mathbf{W} \circ \mathbf{v}\right\|_{p, D ; \mathcal{B}^{r}(\mathcal{X} ; \mathcal{Y})}\|\mathbf{v}\|_{\eta, \infty, D ; \mathcal{X}}^{r}
$$

Proof The Faà di Bruno formula leads to

$$
\begin{aligned}
& \|\mathbf{W} \circ \mathbf{v}\|_{\eta, p, D ; \mathcal{Y}}=\sum_{|\boldsymbol{\alpha}| \leq \eta} \frac{1}{\boldsymbol{\alpha} !}\left\|\partial_{\mathbf{x}}^{\boldsymbol{\alpha}}(\mathbf{W} \circ \mathbf{v})\right\|_{p, D ; \mathcal{Y}} \\
& \leq\|\mathbf{W} \circ \mathbf{v}\|_{p, D ; \mathcal{Y}} \\
& \quad+\sum_{1 \leq|\boldsymbol{\alpha}| \leq \eta} \sum_{r=1}^{|\boldsymbol{\alpha}|} \frac{1}{r !} \sum_{C(\boldsymbol{\alpha}, r)}\left\|\left(D^{r} \mathbf{W} \circ \mathbf{v}\right) \partial_{\mathbf{x}}^{\boldsymbol{\beta}_{1}} \mathbf{v} \cdots \partial_{\mathbf{x}}^{\boldsymbol{\beta}_{r}} \mathbf{v} \prod_{j=1}^{r} \frac{1}{\boldsymbol{\beta}_{j} !}\right\|_{p, D ; \mathcal{Y}}
\end{aligned}
$$

Now, with Lemma 6 ,

$$
\begin{aligned}
& \left\|\left(\mathrm{D}^{r} \mathbf{W} \circ \mathbf{v}\right) \partial_{\mathbf{x}}^{\boldsymbol{\beta}_{1}} \mathbf{v} \cdots \partial_{\mathbf{x}}^{\boldsymbol{\beta}_{r}} \mathbf{v} \prod_{j=1}^{r} \frac{1}{\boldsymbol{\beta}_{j} !}\right\|_{p, D ; \mathcal{Y}} \\
& \quad \leq\left\|\mathrm{D}^{r} \mathbf{W} \circ \mathbf{v}\right\|_{p, D ; \mathcal{B}^{r}(\mathcal{X} ; \mathcal{Y})} \prod_{j=1}^{r} \frac{1}{\boldsymbol{\beta}_{j} !}\left\|\partial_{\mathbf{x}}^{\boldsymbol{\beta}_{j}} \mathbf{v}\right\|_{\infty, D ; \mathcal{X}}
\end{aligned}
$$

leads to

$$
\begin{aligned}
&\|\mathbf{W} \circ \mathbf{v}\|_{\eta, p, D ; \mathcal{Y}} \\
& \leq\|\mathbf{W} \circ \mathbf{v}\|_{p, D ; \mathcal{Y}} \\
&+\sum_{1 \leq|\boldsymbol{\alpha}| \leq \eta} \sum_{r=1}^{|\boldsymbol{\alpha}|} \frac{1}{r !}\left\|\mathrm{D}^{r} \mathbf{W} \circ \mathbf{v}\right\|_{p, D ; \mathcal{B}^{r}(\mathcal{X} ; \mathcal{Y})} \sum_{C(\boldsymbol{\alpha}, r)} \prod_{j=1}^{r} \frac{1}{\boldsymbol{\beta}_{j} !}\left\|\partial_{\mathbf{x}}^{\boldsymbol{\beta}_{j}} \mathbf{v}\right\|_{\infty, D ; \mathcal{X}} \\
& \leq\|\mathbf{W} \circ \mathbf{v}\|_{p, D ; \mathcal{Y}} \\
&+\sum_{r=1}^{\eta} \frac{1}{r !}\left\|\mathrm{D}^{r} \mathbf{W} \circ \mathbf{v}\right\|_{p, D ; \mathcal{B}^{r}(\mathcal{X} ; \mathcal{Y})} \sum_{r \leq|\boldsymbol{\alpha}| \leq \eta} \sum_{C(\boldsymbol{\alpha}, r)} \prod_{j=1}^{r} \frac{1}{\boldsymbol{\beta}_{j} !}\left\|\partial_{\mathbf{x}}^{\boldsymbol{\beta}_{j}} \mathbf{v}\right\|_{\infty, D ; \mathcal{X}} \\
& \leq\|\mathbf{W} \circ \mathbf{v}\|_{p, D ; \mathcal{Y}}+\sum_{r=1}^{\eta} \frac{1}{r !}\left\|\mathrm{D}^{r} \mathbf{W} \circ \mathbf{v}\right\|_{p, D ; \mathcal{B} r(\mathcal{X} ; \mathcal{Y})}\left(\sum_{\boldsymbol{\beta} \leq \boldsymbol{\alpha}} \frac{1}{\boldsymbol{\beta} !}\left\|\partial_{\mathbf{x}}^{\boldsymbol{\beta}} \mathbf{v}\right\|_{\infty, D ; \mathcal{X}}\right)^{r} \\
& \leq \sum_{r=0}^{\eta} \frac{1}{r !}\left\|\mathrm{D}^{r} \mathbf{W} \circ \mathbf{v}\right\|_{p, D ; \mathcal{B}^{r}(\mathcal{X} ; \mathcal{Y})}\|\mathbf{v}\|_{\eta, \infty, D ; \mathcal{X} .}^{r}
\end{aligned}
$$


From Lemma 7, with a second application of the Faà di Bruno formula, see Remark 1, we then can derive the following lemma.

Lemma 8 Let $\eta \in \mathbb{N}, 1 \leq p \leq \infty, \nu \in \mathbb{N}_{f}^{\mathbb{N}^{*}}, \mathcal{X}$ and $\mathcal{Y}$ be Banach spaces,

$$
\mathbf{v}: \square \rightarrow W^{\eta, \infty}(D ; \mathcal{X}),
$$

$X \subset \mathcal{X}$ be open with $\mathrm{img}_{\square} \operatorname{img}_{D} \mathbf{v} \subset X, \mathbf{W}: X \rightarrow \mathcal{Y}$ be $\eta+|\boldsymbol{\nu}|$-times Fréchet differentiable and, for $\boldsymbol{\alpha} \leq \boldsymbol{\nu}$ and $0 \leq t \leq \eta+|\boldsymbol{\nu}|$,

$$
\partial_{\mathbf{y}}^{\boldsymbol{\alpha}} \mathbf{v} \in L_{\mathbb{P}_{\mathbf{y}}}^{\infty}\left(\square ; W^{\eta, \infty}(D ; \mathcal{X})\right), \quad \mathrm{D}^{t} \mathbf{W} \circ \mathbf{v} \in L_{\mathbb{P}_{\mathbf{y}}}^{\infty}\left(\square ; L^{p}\left(D ; \mathcal{B}^{t}(\mathcal{X} ; \mathcal{Y})\right)\right) .
$$

Then, we have

$$
\partial_{\mathbf{y}}^{\boldsymbol{\alpha}}(\mathbf{W} \circ \mathbf{v}) \in L_{\mathbb{P}_{\mathbf{y}}}^{\infty}\left(\square ; W^{\eta, p}(D ; \mathcal{Y})\right)
$$

with

$$
\|\mathbf{W} \circ \mathbf{v}\|_{\eta, p, D ; \mathcal{Y}} \leq \sum_{r=0}^{\eta} \frac{1}{r !}\left\|\mathrm{D}^{r} \mathbf{W} \circ \mathbf{v}\right\|_{p, D ; \mathcal{B}^{r}(\mathcal{X} ; \mathcal{Y})}\|\mathbf{v}\|_{\eta, \infty, D ; \mathcal{X}}^{r}
$$

and, for $\boldsymbol{\alpha} \neq \mathbf{0}$,

$$
\begin{gathered}
\left\|\partial_{\mathbf{y}}^{\boldsymbol{\alpha}}(\mathbf{W} \circ \mathbf{v})\right\|_{\eta, p, D ; \mathcal{Y}} \leq \boldsymbol{\alpha} ! \sum_{s=1}^{|\boldsymbol{\alpha}|} \frac{1}{s !}\left(\sum_{r=0}^{\eta} \frac{1}{r !}\left\|\mathrm{D}^{r+s} \mathbf{W} \circ \mathbf{v}\right\|_{p, D ; \mathcal{B}^{r+s}(\mathcal{X} ; \mathcal{Y})}\|\mathbf{v}\|{ }_{\eta, \infty, D ; \mathcal{X}}^{r}\right) \\
\sum_{C(\boldsymbol{\alpha}, s)} \prod_{j=1}^{s} \frac{1}{\boldsymbol{\beta}_{j} !}\left\|\partial_{\mathbf{y}}^{\boldsymbol{\beta}_{j}} \mathbf{v}\right\|_{\eta, \infty, D ; \mathcal{X}} .
\end{gathered}
$$

Proof The application of Lemma 7 leads to

$$
\begin{aligned}
& \left\|\mathrm{D}^{s} \mathbf{W} \circ \mathbf{v}\right\|_{\eta, p, D ; \mathcal{B}^{s}(\mathcal{X} ; \mathcal{Y})} \\
& =\underset{\mathbf{y} \in \square}{\operatorname{ess} \sup }\left\|\mathrm{D}^{s} \mathbf{W} \circ \mathbf{v}[\mathbf{y}]\right\|_{\eta, p, D ; \mathcal{B}^{s}(\mathcal{X} ; \mathcal{Y})} \\
& \leq \underset{\mathbf{y} \in \square}{\operatorname{ess} \sup } \sum_{r=0}^{\eta} \frac{1}{r !}\left\|\mathrm{D}^{r}\left(\mathrm{D}^{s} \mathbf{W}\right) \circ \mathbf{v}[\mathbf{y}]\right\|_{p, D ; \mathcal{B}^{r}\left(\mathcal{X} ; \mathcal{B}^{s}(\mathcal{X} ; \mathcal{Y})\right)}\|\mathbf{v}[\mathbf{y}]\|_{\eta, \infty, D ; \mathcal{X}}^{r} \\
& \leq \sum_{r=0}^{\eta} \frac{1}{r !}\left\|\mathrm{D}^{r+s} \mathbf{W} \circ \mathbf{v}\right\|_{p, D ; \mathcal{B}^{r+s}(\mathcal{X} ; \mathcal{Y})}\|\mathbf{v}\|_{\eta, \infty, D ; \mathcal{X}}^{r},
\end{aligned}
$$

from which, with $s=0$, the assertion follows directly for $\boldsymbol{\alpha}=\mathbf{0}$.

For $\boldsymbol{\alpha} \neq \mathbf{0}$, we remark that the Faà di Bruno formula yields

$$
\begin{aligned}
& \left\|\partial_{\mathbf{y}}^{\boldsymbol{\alpha}}(\mathbf{W} \circ \mathbf{v})\right\| \|_{\eta, p, D ; \mathcal{Y}} \\
& \quad \leq \boldsymbol{\alpha} ! \sum_{s=1}^{|\boldsymbol{\alpha}|} \frac{1}{s !} \sum_{C(\boldsymbol{\alpha}, s)}\left\|\left(\mathrm{D}^{s} \mathbf{W} \circ \mathbf{v}\right) \partial_{\mathbf{y}}^{\boldsymbol{\beta}_{1}} \mathbf{v} \cdots \partial_{\mathbf{y}}^{\boldsymbol{\beta}_{s}} \mathbf{v} \prod_{j=1}^{s} \frac{1}{\boldsymbol{\beta}_{j} !}\right\| \|_{\eta, p, D ; \mathcal{Y}} \\
& \quad \leq \boldsymbol{\alpha} ! \sum_{s=1}^{|\boldsymbol{\alpha}|} \frac{1}{s !} \sum_{C(\boldsymbol{\alpha}, s)}\left\|\mathrm{D}^{s} \mathbf{W} \circ \mathbf{v}\right\|_{\eta, p, D ; \mathcal{B}}(\mathcal{X} ; \mathcal{Y}) \\
& \quad \prod_{j=1}^{s} \frac{1}{\boldsymbol{\beta}_{j} !}\left\|\partial_{\mathbf{y}}^{\boldsymbol{\beta}_{j}} \mathbf{v}\right\|_{\eta, \infty, D ; \mathcal{X}} \\
& \quad \leq \boldsymbol{\alpha} ! \sum_{s=1}^{|\boldsymbol{\alpha}|} \frac{1}{s !}\left\|\mathrm{D}^{s} \mathbf{W} \circ \mathbf{v}\right\|_{\eta, p, D ; \mathcal{B}^{s}(\mathcal{X} ; \mathcal{Y})} \sum_{C(\boldsymbol{\alpha}, s)} \prod_{j=1}^{s} \frac{1}{\boldsymbol{\beta}_{j} !}\left\|\partial_{\mathbf{y}}^{\boldsymbol{\beta}_{j}} \mathbf{v}\right\|_{\eta, \infty, D ; \mathcal{X}}
\end{aligned}
$$

Note that in the calculation we also use the fact that the submultiplicativity of the norms $\|\cdot\|_{\eta, p, D ; \mathcal{X}}$ shown in Lemma 6 also extends to the norms $\|\cdot\|_{\eta, p, D ; \mathcal{X}}$. 
Now, using these previous results we can consider the regularity of the diffusion coefficient $\mathbf{A}[\mathbf{y}](\mathbf{x}):=\mathbf{D}(\mathbf{V}[\mathbf{y}](\mathbf{x}))$, for which we have the following theorem.

Theorem 2 We know for all $\boldsymbol{\alpha} \in \mathbb{N}_{f}^{\mathbb{N}^{*}}$ that

$$
\left\|\partial_{\mathbf{y}}^{\boldsymbol{\alpha}} \mathbf{A}\right\|_{\tau, \infty, D ; \mathbb{R}_{\mathrm{symm}}^{d \times d}} \leq|\boldsymbol{\alpha}| ! k_{\tau, \mathbf{A}} \boldsymbol{\mu}_{\tau}^{\boldsymbol{\alpha}}
$$

where $\boldsymbol{\mu}_{\tau}=\left(\mu_{\tau, k}\right)_{k \in \mathbb{N}^{*}}$ with

$$
k_{\tau, \mathbf{A}}:=(\tau+1) k_{\mathbf{D}} 2^{\tau} c_{\mathbf{D}}^{\tau} c_{\boldsymbol{\gamma}_{\tau}}^{\tau} \quad \text { and } \quad \mu_{\tau, k}:=\frac{2 c_{\mathbf{D}} c_{\boldsymbol{\gamma}_{\tau}}}{\log 2} \gamma_{\tau, k}
$$

Proof Firstly, since $\mathbf{V}$ depends affinely on $\mathbf{y}$ we know that

$$
\left\|\partial_{\mathbf{y}}^{\boldsymbol{\alpha}} \mathbf{V}\right\|_{\tau, \infty, D ; \mathbb{R}^{d}} \leq c_{\boldsymbol{\gamma}_{\tau}} \boldsymbol{\gamma}_{\tau}^{\boldsymbol{\alpha}}
$$

Moreover, as $\mathbf{V}[\mathbf{y}](\mathbf{x})$ almost surely and almost everywhere lies in $V$, Theorem 1 yields

$$
\left\|\mathrm{D}^{r} \mathbf{D} \circ \mathbf{V}\right\|_{\infty, D ; \mathcal{B}^{t}\left(\mathbb{R}^{d} ; \mathbb{R}_{\mathrm{symm}}^{d \times d}\right)} \leq r ! k_{\mathbf{D}} c_{\mathbf{D}}^{r} .
$$

With this, because $\mathbf{A}=\mathbf{D} \circ \mathbf{V}$, we can employ Lemma 8 to arrive at

$$
\begin{aligned}
\|\mathbf{A}\|_{\tau, \infty, D ; \mathbb{R}_{\text {symm }}^{d \times d}} & \leq \sum_{r=0}^{\eta} \frac{1}{r !}\left\|\mathrm{D}^{r} \mathbf{D} \circ \mathbf{V}\right\|_{\left.\infty, D ; \mathcal{B}^{r}\left(\mathbb{R}^{d} ; \mathbb{R}_{\mathrm{symm}}^{d \times d}\right)\right)}\|\mathbf{V}\|_{\eta, \tau, D ; \mathbb{R}^{d}}^{r} \\
& \leq \sum_{r=0}^{\tau} \frac{1}{r !} r ! k_{\mathbf{D}} c_{\mathbf{D}^{r}}^{r} c_{\gamma_{\tau}}^{r} \\
& \leq(\tau+1) k_{\mathbf{D}} c_{\mathbf{D}}^{\tau} c_{\gamma_{\tau}}^{\tau}
\end{aligned}
$$

as well as, for $\boldsymbol{\alpha} \neq \mathbf{0}$,

$$
\begin{aligned}
& \left\|\partial_{\mathbf{y}}^{\boldsymbol{\alpha}} \mathbf{A}\right\|_{\tau, \infty, D ; \mathbb{R}_{\text {symm }}^{d \times d}} \\
& \leq \boldsymbol{\alpha} ! \sum_{s=1}^{|\boldsymbol{\alpha}|} \frac{1}{s !}\left(\sum_{r=0}^{\tau} \frac{1}{r !}\left\|\mathrm{D}^{r+s} \mathbf{D} \circ \mathbf{V}\right\|_{\infty, D ; \mathcal{B}^{r+s}\left(\mathbb{R}^{d} ; \mathbb{R}_{\mathrm{symm}}^{d \times d}\right)}\|\mathbf{V}\|_{\tau, \infty, D ; \mathbb{R}^{d}}^{r}\right) \\
& \sum_{C(\boldsymbol{\alpha}, s)} \prod_{j=1}^{s} \frac{1}{\boldsymbol{\beta}_{j} !}\left\|\partial_{\mathbf{y}}^{\boldsymbol{\beta}_{j}} \mathbf{V}\right\|_{\tau, \infty, D ; \mathbb{R}^{d}} \\
& \leq \boldsymbol{\alpha} ! \sum_{s=1}^{|\boldsymbol{\alpha}|} \frac{1}{s !}\left(\sum_{r=0}^{\tau} \frac{1}{r !}(r+s) ! k_{\mathbf{D}} c_{\mathbf{D}}^{r+s} c_{\boldsymbol{\gamma}_{\tau}}^{r}\right) \sum_{C(\boldsymbol{\alpha}, s)} \prod_{j=1}^{s} \frac{1}{\boldsymbol{\beta}_{j} !} c_{\boldsymbol{\gamma}_{\tau}} \boldsymbol{\gamma}_{\tau}^{\boldsymbol{\beta}_{j}} \\
& \leq \boldsymbol{\alpha} !(\tau+1) k_{\mathbf{D}} 2^{\tau} c_{\mathbf{D}}^{\tau} c_{\boldsymbol{\gamma}_{\tau}}^{\tau} 2^{|\boldsymbol{\alpha}|} c_{\mathbf{D}}^{|\boldsymbol{\alpha}|}\left(\sum_{s=1}^{|\boldsymbol{\alpha}|} \sum_{C(\boldsymbol{\alpha}, s)} \prod_{j=1}^{s} \frac{1}{\boldsymbol{\beta}_{j} !}\right) c_{\boldsymbol{\gamma}_{\tau} \mid}^{|\boldsymbol{\alpha}|} \boldsymbol{\gamma}_{\tau}^{\boldsymbol{\alpha}}
\end{aligned}
$$

Now, since it follows from [10] that

$$
\boldsymbol{\alpha} ! \sum_{C(\boldsymbol{\alpha}, s)} \prod_{j=1}^{s} \frac{1}{\boldsymbol{\beta}_{j} !}=s ! S_{|\boldsymbol{\alpha}|, s},
$$


where $S_{n, s}$ denotes the Stirling numbers of the second kind, see [1] and since we know that $\sum_{s=1}^{|\boldsymbol{\alpha}|} s ! S_{|\boldsymbol{\alpha}|, s}$ equals the $|\boldsymbol{\alpha}|$-th ordered Bell number, we have the following bound

$$
\boldsymbol{\alpha} ! \sum_{s=1}^{|\boldsymbol{\alpha}|} \sum_{C(\boldsymbol{\alpha}, s)} \prod_{j=1}^{s} \frac{1}{\boldsymbol{\beta}_{j} !}=\sum_{s=1}^{|\boldsymbol{\alpha}|} s ! S_{|\boldsymbol{\alpha}|, s} \leq \frac{|\boldsymbol{\alpha}| !}{(\log 2)^{|\boldsymbol{\alpha}|}}
$$

see [4]. Thus, combining these estimates finally yields the assertions.

Now, Assumption 2 directly implies the following result.

Lemma 9 The unique solution $u \in L_{\mathbb{P}_{\mathbf{y}}}^{\infty}\left(\square ; H_{0}^{1}(D)\right)$ of $(7)$ moreover also fulfils $u \in L_{\mathbb{P}_{\mathbf{y}}}^{\infty}\left(\square ; H^{\tau+1}(D)\right)$, with

$$
\|u\|_{\tau+1,2, D} \leq c_{\tau}\|f\|_{\tau-1,2, D}
$$

where

$$
c_{\tau}:=\max _{0 \leq s \leq\|\mathbf{A}\|_{\mathcal{R}_{\tau}}} C_{\tau, e r}(D, \underline{a}, s) .
$$

However, by also leveraging the higher spatial regularity in the Karhunen-Loève expansion of the random vector-valued field, we can show, with the usual bootstrapping argument, that the solution $u$ admits analytic regularity with respect to the stochastic parameter $\mathbf{y}$ also in the $H^{\tau+1}(D)$-norm. This mixed regularity is then the essential ingredient when applying multilevel methods.

Theorem 3 The derivatives in $\mathbf{y}$ of the solution $u$ of (7) satisfy

$$
\left\|\partial_{\mathbf{y}}^{\boldsymbol{\alpha}} u\right\|_{\tau+1,2, D} \leq|\boldsymbol{\alpha}| ! c^{|\boldsymbol{\alpha}|+1} \boldsymbol{\mu}_{\tau}^{\boldsymbol{\alpha}}
$$

where

$$
c:=\max \left\{2,2 c_{\tau} \tau^{2} d^{2} k_{\tau, \mathbf{A}}, c_{\tau}\|f\|_{\tau-1,2, D}\right\}
$$

Proof By differentiation of the variational formulation (7) with respect to $\mathbf{y}$ we arrive, for arbitrary $v \in H_{0}^{1}(D)$, at

$$
\left(\partial_{\mathbf{y}}^{\boldsymbol{\alpha}}\left(\mathbf{A} \nabla_{\mathbf{x}} u\right), \nabla_{\mathbf{x}} v\right)_{L^{2}\left(D ; \mathbb{R}^{d}\right)}=0 .
$$

Applying the Leibniz rule on the left-hand side yields

$$
\left(\sum_{\boldsymbol{\beta} \leq \boldsymbol{\alpha}}\left(\begin{array}{c}
\boldsymbol{\alpha} \\
\boldsymbol{\beta}
\end{array}\right) \partial_{\mathbf{y}}^{\boldsymbol{\alpha}-\boldsymbol{\beta}} \mathbf{A} \partial_{\mathbf{y}}^{\boldsymbol{\beta}} \nabla_{\mathbf{x}} u, \nabla_{\mathbf{x}} v\right)_{L^{2}\left(D ; \mathbb{R}^{d}\right)}=0
$$

Then, by rearranging and using the linearity of the gradient, we find

$$
\left(\mathbf{A} \nabla_{\mathbf{x}} \partial_{\mathbf{y}}^{\boldsymbol{\alpha}} u, \nabla_{\mathbf{x}} v\right)_{L^{2}\left(D ; \mathbb{R}^{d}\right)}=-\left(\sum_{\boldsymbol{\beta}<\boldsymbol{\alpha}}\left(\begin{array}{l}
\boldsymbol{\alpha} \\
\boldsymbol{\beta}
\end{array}\right) \partial_{\mathbf{y}}^{\boldsymbol{\alpha}-\boldsymbol{\beta}} \mathbf{A} \nabla_{\mathbf{x}} \partial_{\mathbf{y}}^{\boldsymbol{\beta}} u, \nabla_{\mathbf{x}} v\right)_{L^{2}\left(D ; \mathbb{R}^{d}\right)} .
$$

Using Green's identity, we can then write

$$
\left(\mathbf{A} \nabla_{\mathbf{x}} \partial_{\mathbf{y}}^{\boldsymbol{\alpha}} u, \nabla_{\mathbf{x}} v\right)_{L^{2}\left(D ; \mathbb{R}^{d}\right)}=\left(\sum_{\boldsymbol{\beta}<\boldsymbol{\alpha}}\left(\begin{array}{c}
\boldsymbol{\alpha} \\
\boldsymbol{\beta}
\end{array}\right) \operatorname{div}_{\mathbf{x}}\left(\partial_{\mathbf{y}}^{\boldsymbol{\alpha}-\boldsymbol{\beta}} \mathbf{A} \nabla_{\mathbf{x}} \partial_{\mathbf{y}}^{\boldsymbol{\beta}} u\right), v\right)_{L^{2}(D ; \mathbb{R})}
$$


Thus, we arrive at

$$
\begin{aligned}
\left\|\partial_{\mathbf{y}}^{\boldsymbol{\alpha}} u\right\|_{\tau+1,2, D} & \leq c_{\tau} \sum_{\boldsymbol{\beta}<\boldsymbol{\alpha}}\left(\begin{array}{c}
\boldsymbol{\alpha} \\
\boldsymbol{\beta}
\end{array}\right)\left\|\operatorname{div}_{\mathbf{x}}\left(\partial_{\mathbf{y}}^{\boldsymbol{\alpha}-\boldsymbol{\beta}} \mathbf{A} \nabla_{\mathbf{x}} \partial_{\mathbf{y}}^{\boldsymbol{\beta}} u\right)\right\|_{\tau-1,2, D} \\
& \leq c_{\tau} \sum_{\boldsymbol{\beta}<\boldsymbol{\alpha}}\left(\begin{array}{c}
\boldsymbol{\alpha} \\
\boldsymbol{\beta}
\end{array}\right) \tau d\left\|\partial_{\mathbf{y}}^{\boldsymbol{\alpha}-\boldsymbol{\beta}} \mathbf{A} \nabla_{\mathbf{x}} \partial_{\mathbf{y}}^{\boldsymbol{\beta}} u\right\|_{\tau, 2, D} \\
& \leq c_{\tau} \sum_{\boldsymbol{\beta}<\boldsymbol{\alpha}}\left(\begin{array}{c}
\boldsymbol{\alpha} \\
\boldsymbol{\beta}
\end{array}\right) \tau d\left\|\partial_{\mathbf{y}}^{\boldsymbol{\alpha}-\boldsymbol{\beta}} \mathbf{A}\right\|\left\|_{\tau, \infty, D}\right\| \nabla_{\mathbf{x}} \partial_{\mathbf{y}}^{\boldsymbol{\beta}} u \|_{\tau, 2, D} \\
& \leq c_{\tau} \tau^{2} d^{2} k_{\tau, \mathbf{A}} \sum_{\boldsymbol{\beta}<\boldsymbol{\alpha}}\left(\begin{array}{c}
\boldsymbol{\alpha} \\
\boldsymbol{\beta}
\end{array}\right)|\boldsymbol{\alpha}-\boldsymbol{\beta}| ! \boldsymbol{\mu}_{\tau}^{\boldsymbol{\alpha}-\boldsymbol{\beta}}\left\|\partial_{\mathbf{y}}^{\boldsymbol{\beta}} u\right\|_{\tau+1,2, D},
\end{aligned}
$$

by using Lemma 5 and the submultiplicativity of the norms $\|\cdot\|_{\eta, p, D ; \mathcal{X}}$, which follows from Lemma 6 , as well as the bounds from Lemma 2. From this we then arrive at

$$
\left\|\partial_{\mathbf{y}}^{\boldsymbol{\alpha}} u\right\|_{\tau+1,2, D} \leq \frac{c}{2} \sum_{\boldsymbol{\beta}<\boldsymbol{\alpha}}\left(\begin{array}{c}
\boldsymbol{\alpha} \\
\boldsymbol{\beta}
\end{array}\right)|\boldsymbol{\alpha}-\boldsymbol{\beta}| ! \boldsymbol{\mu}_{\tau}^{\boldsymbol{\alpha}-\boldsymbol{\beta}}\left\|\partial_{\mathbf{y}}^{\boldsymbol{\beta}} u\right\|_{\tau+1,2, D}
$$

We note that, by definition of $c$, we have $c \geq 2$ and furthermore, because of Lemma 9, we also have that $\|u\|_{H^{1}(D)} \leq c$, which means that the assertion is true for $|\boldsymbol{\alpha}|=0$. Thus, we can use an induction over $|\boldsymbol{\alpha}|$ to prove the hypothesis

$$
\left\|\partial_{\mathbf{y}}^{\boldsymbol{\alpha}} u\right\|_{\tau+1,2, D} \leq|\boldsymbol{\alpha}| ! \boldsymbol{\mu}_{\tau}^{\boldsymbol{\alpha}} c^{|\boldsymbol{\alpha}|+1}
$$

for $|\boldsymbol{\alpha}|>0$.

Let the assertions hold for all $\boldsymbol{\alpha}$, which satisfy $|\boldsymbol{\alpha}| \leq n-1$ for some $n \geq 1$. Then, we know for all $\boldsymbol{\alpha}$ with $|\boldsymbol{\alpha}|=n$ that

$$
\begin{aligned}
\left\|\partial_{\mathbf{y}}^{\boldsymbol{\alpha}} u\right\|_{\tau+1,2, D} & \leq \frac{c}{2} \sum_{\boldsymbol{\beta}<\boldsymbol{\alpha}}\left(\begin{array}{c}
\boldsymbol{\alpha} \\
\boldsymbol{\beta}
\end{array}\right)|\boldsymbol{\alpha}-\boldsymbol{\beta}| ! \boldsymbol{\mu}_{\tau}^{\boldsymbol{\alpha}-\boldsymbol{\beta}}\left\|\partial_{\mathbf{y}}^{\boldsymbol{\beta}} u\right\|_{\tau+1,2, D} \\
& \leq \frac{c}{2} \boldsymbol{\mu}_{\tau}^{\boldsymbol{\alpha}} \sum_{\boldsymbol{\beta}<\boldsymbol{\alpha}}\left(\begin{array}{c}
\boldsymbol{\alpha} \\
\boldsymbol{\beta}
\end{array}\right)|\boldsymbol{\alpha}-\boldsymbol{\beta}| !|\boldsymbol{\beta}| ! c^{|\boldsymbol{\beta}|+1} \\
& =\frac{c}{2} \boldsymbol{\mu}_{\tau}^{\boldsymbol{\alpha}} \sum_{j=0}^{n-1} \sum_{\substack{\boldsymbol{\beta}<\boldsymbol{\alpha} \\
|\boldsymbol{\beta}|=j}}\left(\begin{array}{c}
\boldsymbol{\alpha} \\
\boldsymbol{\beta}
\end{array}\right)|\boldsymbol{\alpha}-\boldsymbol{\beta}| !|\boldsymbol{\beta}| ! c^{|\boldsymbol{\beta}|+1}
\end{aligned}
$$

Making use of the combinatorial identity

$$
\sum_{\substack{\boldsymbol{\beta} \leq \boldsymbol{\alpha} \\
|\boldsymbol{\beta}|=j}}\left(\begin{array}{c}
\boldsymbol{\alpha} \\
\boldsymbol{\beta}
\end{array}\right)=\left(\begin{array}{c}
|\boldsymbol{\alpha}| \\
j
\end{array}\right)
$$


yields

$$
\begin{aligned}
\left\|\partial_{\mathbf{y}}^{\boldsymbol{\alpha}} u\right\|_{\tau+1,2, D} & \leq \frac{c}{2} \boldsymbol{\mu}_{\tau}^{\alpha} \sum_{j=0}^{n-1}\left(\begin{array}{c}
|\boldsymbol{\alpha}| \\
j
\end{array}\right)(|\boldsymbol{\alpha}|-j) ! j ! c^{j+1}=\frac{c}{2}|\boldsymbol{\alpha}| ! \boldsymbol{\mu}_{\tau}^{\boldsymbol{\alpha}} c \sum_{j=0}^{n-1} c^{j} \\
& \leq \frac{c}{2(c-1)}|\boldsymbol{\alpha}| ! \boldsymbol{\mu}_{\tau}^{\boldsymbol{\alpha}} c^{|\boldsymbol{\alpha}|+1} .
\end{aligned}
$$

Now, since $c \geq 2$, we have $c \leq 2(c-1)$ and hence also

$$
\left\|\partial_{\mathbf{y}}^{\boldsymbol{\alpha}} u\right\|_{\tau+1,2, D} \leq|\boldsymbol{\alpha}| ! \boldsymbol{\mu}_{\tau}^{\boldsymbol{\alpha}} c^{|\boldsymbol{\alpha}|+1} .
$$

This completes the proof.

3.3 Numerical quadrature in the parameter

We now impose some common assumptions, which make the Karhunen-Loève expansion computationally feasible.

Assumption 3 The random variables $\left(y_{k}\right)_{k \in \mathbb{N}^{*}}$ are independent and identically distributed. Moreover, they are uniformly distributed on $[-1,1]$.

By rescaling the $\sigma_{k}$ with the factor 2 we thus can replace the choice $\square:=\mathbb{R}^{\mathbb{N}^{*}}$ with $\square:=\left[-\frac{1}{2}, \frac{1}{2}\right]^{\mathbb{N}^{*}} \cdot{ }^{2}$

Coming from the solution of (7), that is $u \in L_{\mathbb{P}_{\mathbf{y}}}^{\infty}\left(\square ; H^{\tau+1}(D)\right)$, we now wish to know the moments of $u$. In this section, we will therefore consider the approximation of the mean of $u$.

The mean of $u$ is given by the Bochner integral

$$
\mathbb{E}[u](\mathbf{x})=\int_{\square} u[\mathbf{y}](\mathbf{x}) \mathrm{d} \mathbf{y} .
$$

Therefore, we may proceed to approximate it by considering a generic quadrature method $Q_{N}$; that is

$$
\mathbb{E}[u](\mathbf{x}) \approx Q_{N}[u](\mathbf{x}):=\sum_{i=1}^{N} \omega_{i}^{(N)} u\left(\mathbf{x}, \boldsymbol{\xi}_{i}^{(N)}\right),
$$

where

$$
\left\{\left(\omega_{i}^{(N)}, \boldsymbol{\xi}_{i}^{(N)}\right)\right\}_{i=1}^{N} \subset \mathbb{R} \times\left[-\frac{1}{2}, \frac{1}{2}\right]^{\mathbb{N}^{*}}
$$

are the weight and evaluation point pairs. We assume that the quadrature chosen fulfils

$$
\left\|\mathbb{E}[u]-Q_{N}[u]\right\|_{1,2, D} \leq c C\left(c \boldsymbol{\mu}_{\tau}\right) N^{-r}
$$

for some constants $C\left(c \boldsymbol{\mu}_{\tau}\right)=C>0$ and $r>0$.

${ }^{2}$ Clearly, in practice the Karhunen-Loève expansion also has to be truncated after the first $M$ summands, for some $M \in \mathbb{N}$. However, the Taylor expansion of $u$ at the point $\mathbf{0}$ and the bounds from Theorem 3 imply that the error incurred by such a truncation tends to 0 as $M$ tends to infinity. Thus, a large enough $M$ can always be choosen to give the desired accuracy, while, as the quadrature considered has constants that do not depend on $M$, increasing the $M$ also does not deteriorate the accuracy of the quadrature error. 
We will employ the quasi-Monte Carlo quadrature based on the Halton sequence, i.e. $\omega_{i}^{(N)}=1 / N$ and $\boldsymbol{\xi}_{i}^{(N)}=\chi_{i}-\frac{1}{2} \mathbf{1}$, where $\chi_{i}$ denotes the $i$-th $M$ dimensional Halton point, cf. [18]. Then, we know that, given that there exists an $\varepsilon>0$ such that $\mu_{\tau, k} \lesssim k^{-3-\varepsilon}$ holds, for every $\delta>0$ there exists a constant $C=C(\delta)>0$ such that (9) holds for $r=1-\delta$, see e.g. [22] which is a consequence of [36]. Clearly, other, possibly more sophisticated, quadrature methods may also be considered, for example, other quasi-Monte Carlo quadratures, such as those based on the Sobol sequence or other low-discrepancy sequences as well as their higher-order adaptations, and anisotropic sparse grid quadratures, see e.g. $[12,17$, $30,34]$.

To approximate the mean of $u$ as in (8), we require the values $u[\mathbf{y}]$ for $\mathbf{y}=\boldsymbol{\xi}_{i}$. These values can be approximated by $u_{l}[\mathbf{y}]$, where $u_{l}$ is the Galerkin approximation of the spatially weak formulation on a finite dimensional subspace $V_{l}$ of $H_{0}^{1}(D)$; that is, $u_{l}$ is the solution of

$$
\left\{\begin{array}{c}
\text { Find } u_{l} \in L_{\mathbb{P}_{\mathbf{y}}}^{\infty}\left(\square ; V_{l}\right) \text { such that } \\
\left(\mathbf{A}[\mathbf{y}] \nabla_{\mathbf{x}} u_{l}[\mathbf{y}], \nabla_{\mathbf{x}} v\right)_{L^{2}\left(D ; \mathbb{R}^{d}\right)}=(f, v)_{L^{2}\left(D ; \mathbb{R}^{d}\right)} \\
\text { for almost every } \mathbf{y} \in \square \text { and all } v \in V_{l} .
\end{array}\right.
$$

We assume that a sequence of $V_{l}$ can be chosen for $l \in \mathbb{N}$ such that there is a constant $K$ with

$$
\left\|u-u_{l}\right\|_{1,2, D} \leq K\|u\|_{\tau+1,2, D} 2^{-\tau l} .
$$

For example, we can consider $V_{l}$ to be the spaces of continuous finite elements of order $\tau$ coming from a sequence of quasi-uniform meshes $\mathcal{T}_{l}$ using isoparametric elements, where the mesh size behaves like $2^{-l}$. Then, this is known from finite element theory, see e.g. $[5,6]$.

The combination of the error estimates (9) and (10) then leads to

$$
\begin{aligned}
\left\|\mathbb{E}[u]-Q_{N}\left[u_{l}\right]\right\|_{1,2, D} & \leq\left\|\mathbb{E}[u]-Q_{N}[u]\right\|_{1,2, D}+\left\|Q_{N}\left[u-u_{l}\right]\right\|_{1,2, D} \\
& \leq c C\left(c \boldsymbol{\mu}_{\tau}\right) N^{-r}+K\|u\|_{\tau+1,2, D} 2^{-\tau l} .
\end{aligned}
$$

Thus, choosing $N_{l}:=\left\lceil 2^{\tau l / r}\right\rceil$ finally yields

$$
\left\|\mathbb{E}[u]-Q_{N_{l}}\left[u_{l}\right]\right\|_{1,2, D} \lesssim 2^{-\tau l} .
$$

In contrast, the mixed regularity, shown before in Theorem 3, allows us to consider a multilevel adaptation, which may be given as

$$
\mathbb{E}[u] \approx Q_{l}^{\mathrm{ML}}\left[u_{0}, \ldots, u_{l}\right]:=\sum_{k=0}^{l} \Delta Q_{k}\left[u_{l-k}\right]
$$

where $\Delta Q_{0}:=Q_{N_{0}}$ and $\Delta Q_{k}:=Q_{N_{k}}-Q_{N_{k-1}}$. Indeed, this is the sparse grid combination technique as introduced in [15], see also [14,20,23]. As Theorem 3 yields an analogous result to [23, Lemma 8.1], it thus follows that

$$
\left\|\mathbb{E}[u]-Q_{l}^{\mathrm{ML}}\left[u_{0}, \ldots, u_{l}\right]\right\|_{1,2, D} \lesssim l 2^{-\tau l} .
$$

For complexity considerations, we shall consider a quadrature that is nested, i.e. a quadrature method were increasing the number of evaluation points from $N$ 
to $N+1$ is done by adding one further evaluation point to the set of evaluation points one already had. Thus, we may set $\boldsymbol{\xi}_{i}=\boldsymbol{\xi}_{i}^{(N)}$ as it does not depend on $N$. Then, we note that $Q_{l}^{\mathrm{ML}}\left[u_{0}, \ldots, u_{l}\right]$ may explicitly be stated as

$$
\begin{aligned}
Q_{l}^{\mathrm{ML}}\left[u_{0}, \ldots, u_{l}\right](\mathbf{x})= & \sum_{i=1}^{N_{0}} \omega_{i}^{\left(N_{0}\right)} u_{l}\left(\mathbf{x}, \boldsymbol{\xi}_{i}\right) \\
& +\sum_{k=1}^{l}\left(\sum_{i=1}^{N_{k-1}}\left(\omega_{i}^{\left(N_{k}\right)}-\omega_{i}^{\left(N_{k-1}\right)}\right) u_{l-k}\left(\mathbf{x}, \boldsymbol{\xi}_{i}\right)\right. \\
& \left.+\sum_{i=N_{k-1}+1}^{N_{k}} \omega_{i}^{\left(N_{k}\right)} u_{l-k}\left(\mathbf{x}, \boldsymbol{\xi}_{i}\right)\right)
\end{aligned}
$$

Computing $Q_{N_{l}}\left[u_{l}\right]$ requires thus the values $u_{l, i}(\mathbf{x}):=u\left(\mathbf{x}, \boldsymbol{\xi}_{i}\right)$, which can be derived by solving

$$
\left\{\begin{array}{l}
\text { Find } u_{l, i} \in V_{l} \text { such that } \\
\quad\left(\mathbf{A}\left(\boldsymbol{\xi}_{i}\right) \nabla_{\mathbf{x}} u_{l, i}, \nabla_{\mathbf{x}} v\right)_{L^{2}\left(D ; \mathbb{R}^{d}\right)}=(f, v)_{L^{2}\left(D ; \mathbb{R}^{d}\right)} \quad \text { for all } v \in V_{l} .
\end{array}\right.
$$

Generally, when considering a sequence of finite element spaces $V_{l}$ as described above, the number of degrees of freedom behaves like $\mathcal{O}\left(2^{l d}\right)$ and computing one $u_{l, i}$ using state of the art methods will have a complexity that is $\mathcal{O}\left(2^{l d}\right)$.

Therefore, the computation of the singlelevel quadrature $Q_{N_{l}}\left[u_{l}\right]$ has a complexity of $\mathcal{O}\left(2^{l(\tau / r+d)}\right)$. However, for the computation of the multilevel quadrature $Q_{l}^{\mathrm{ML}}\left[u_{0}, \ldots, u_{l}\right]$, we arrive at an over-all complexity of

$$
\sum_{k=0}^{l} \sum_{i=1}^{N_{k}} \mathcal{O}\left(2^{(l-k) d}\right)=\sum_{k=0}^{l} \mathcal{O}\left(2^{k \tau / r} 2^{(l-k) d}\right)= \begin{cases}\mathcal{O}\left(2^{l \tau / r}\right) & \text { for } d<\tau / r \\ \mathcal{O}\left(l 2^{l d}\right) & \text { for } d=\tau / r \\ \mathcal{O}\left(2^{l d}\right) & \text { for } d>\tau / r\end{cases}
$$

We mention that also non-nested quadrature formulae can be used but lead to a somewhat larger constant in the complexity estimate, see [14] for the details.

Remark 2 The singlelevel approach gives an error scaling like $\left(2^{\tau}\right)^{l}$ with a complexity scaling like $\left(2^{\tau / r+d}\right)^{l}$. The multilevel approach, however, for any choosen $\varepsilon>0$, yields an error scaling like $l\left(2^{-\tau}\right)^{l} \lesssim\left(2^{-\tau+\varepsilon}\right)^{l}$ with a complexity of $\left(2^{\tau / r}\right)^{l}$, $l\left(2^{d}\right)^{l} \lesssim\left(2^{d+\varepsilon}\right)^{l}$ or $\left(2^{d}\right)^{l}$, respectively. Thus, by considering a small $\varepsilon>0$ we see that the multilevel approach has an error to complexity scaling that essentially either is the error to complexity scaling of the quadrature method or that of the space discretisation.

Remark 3 If we redefine the $N_{l}$ as $N_{l}:=\left\lceil l^{(1+\varepsilon) / r} 2^{\tau l / r}\right\rceil$ for an $\varepsilon>0$, as proposed in [2], then we arrive at

$$
\left\|\mathbb{E}[u]-Q_{l}^{\mathrm{ML}}\left[u_{0}, \ldots, u_{l}\right]\right\|_{H^{1}(D)} \lesssim 2^{-\tau l} .
$$

So, the logarithmic factor, which shows up in the convergence rate, can be removed by increasing the quadrature accuracy slightly faster. Note that this modification increases the hidden constant with a dependance on $\varepsilon$. 
In the particular situation of the quasi-Monte Carlo method based on the Halton sequence, we can consider $\delta^{\prime}$ such that $\delta>\delta^{\prime}>0$. Then, with a similar argument as in [2], it follows that

$$
\left\|\mathbb{E}[u]-Q_{l}^{\mathrm{ML}}\left[u_{0}, \ldots, u_{l}\right]\right\|_{H^{1}(D)} \lesssim 2^{-\tau l} .
$$

That is, the logarithmic factor, which shows up in the convergence rate, is removed, while the hidden constant increases with a dependance on $\delta^{\prime}$.

While we have exclusively considered the case of the mean of the solution $u$ here, we do note that analogous statements may also be shown for example for the higher-order moments, see [20,23] for instance.

\section{Numerical Results}

We will now consider two examples of the model problem (1) with a diffusion coefficient of form (2) using the unit cube $D:=(0,1)^{3}$ as the domain of computations. Therefore, in view of $H^{2}$-regularity of the spatial problem under consideration, we are only considering the situation with $\tau=1$. In both examples, we set the global strength $a$ to $a:=0.12$ and choose the right hand side $f \equiv 1$. For convenience, we define

$$
s_{j}\left(\mathbf{x}, \mathbf{x}^{\prime}\right):=16 \cdot x_{j}\left(1-x_{j}\right) \cdot x_{j}^{\prime}\left(1-x_{j}^{\prime}\right) .
$$

Example 1 In this first example, we choose the description of $\mathbf{V}$ to be defined by

$$
\mathbb{E}[\mathbf{V}](\mathbf{x}):=\left[\begin{array}{lll}
1 & 0 & 0
\end{array}\right]^{\top}
$$

and

$$
\operatorname{Cov}[\mathbf{V}]\left(\mathbf{x}, \mathbf{x}^{\prime}\right):=\frac{1}{100} \exp \left(-\frac{\left\|\mathbf{x}-\mathbf{x}^{\prime}\right\|_{2}^{2}}{50}\right)\left[\begin{array}{ccc}
1 & 0 & 0 \\
0 & 9 s_{2}\left(\mathbf{x}, \mathbf{x}^{\prime}\right) & 0 \\
0 & 0 & 9 s_{3}\left(\mathbf{x}, \mathbf{x}^{\prime}\right)
\end{array}\right]
$$

We note that for $j \in\{2,3\}$ the covariance in the normal direction on the parts of the boundary with $x_{j} \in\{0,1\}$ is suppressed.

In Figure 1 the stream traces of two samples of the vector field $\mathbf{V} /\|\mathbf{V}\|_{2}$ are shown. By definition of the mapping $\mathbf{D}$ these stream traces show the orientation of the fibres which are described by $\mathbf{D} \circ \mathbf{V}$. They are coloured according to $\|\mathbf{V}\|_{2}$, which by the mapping $\mathbf{D}$ encodse the diffusion strength in the fiber direction.

Example 2 For this second example we choose the description of $\mathbf{V}$ to be defined by

$$
\mathbb{E}[\mathbf{V}](\mathbf{x}):=\left[\begin{array}{c}
\cos \left(\left(x_{3}-0.5\right) \frac{\pi}{3}\right) \\
\sin \left(\left(x_{3}-0.5\right) \frac{\pi}{3}\right) \\
0
\end{array}\right]
$$

and

$$
\operatorname{Cov}[\mathbf{V}]\left(\mathbf{x}, \mathbf{x}^{\prime}\right):=\frac{9}{100} \exp \left(-\frac{\left\|\mathbf{x}-\mathbf{x}^{\prime}\right\|_{2}^{2}}{50}\right)\left[\begin{array}{ccc}
s_{1}\left(\mathbf{x}, \mathbf{x}^{\prime}\right) & 0 & 0 \\
0 & s_{2}\left(\mathbf{x}, \mathbf{x}^{\prime}\right) & 0 \\
0 & 0 & s_{3}\left(\mathbf{x}, \mathbf{x}^{\prime}\right)
\end{array}\right] .
$$

Here, the covariance in the normal direction on all of the boundary is suppressed. 

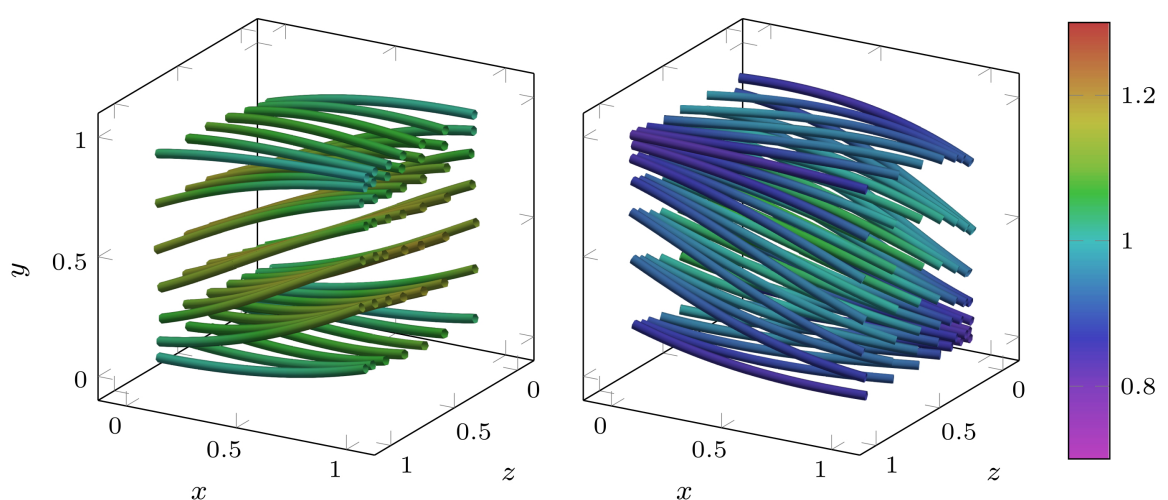

Fig. 1 Example 1. Fibre visualisations for two samples of the parameter $\mathbf{y}$.

The numerical implementation is performed with aid of the problem-solving environment DOLFIN [29], which is a part of the FEniCS Project [29]. The Karhunen-Loève expansion of the vector field $\mathbf{V}$ is computed by the pivoted Cholesky decomposition, see $[19,21]$ for the details. For the finite element discretisation, we employ the sequence of nested triangulations $\mathcal{T}_{l}$, yielded by successive uniform refinement, i.e. cutting each tetrahedron into 8 tetrahedra. The base triangulation $\mathcal{T}_{0}$ consists of $6 \cdot 2^{3}=48$ tetrahedra. Then, we use interpolation with continuous element-wise linear functions and the truncated pivoted Cholesky decomposition for the Karhunen-Loève expansion approximation and continuous element-wise linear functions in space. The truncation criterion for the pivoted Cholesky decomposition is that the relative trace error is smaller than $10^{-4} \cdot 4^{-l}$.

Since the exact solutions of the examples are unknown, the errors will have to be estimated. Therefore, in this section, we will estimate the errors for the levels 0 to 5 by substituting the exact solution with the approximate solution computed on the level 6 triangulation $\mathcal{T}_{6}$ using the quasi-Monte Carlo quadrature based on Halton points with $10^{4}$ samples.

For every level, we also define the number of samples used by the quasi-Monte Carlo method based on Halton points (QMC); we choose

$$
N_{l}:=\left\lceil 2^{l /(1-\delta)} \cdot 10\right\rceil
$$

with $\delta:=0.2$; see Table 1 for the resulting values of $N_{l}$. This then also implies the amount of samples used on the different levels when using the multilevel quasiMonte Carlo method based on Halton points (MLQMC). Based on these choices, we expect to see an asymptotic rate of convergence of $2^{-l}$ in the $H^{1}$-norm for the mean and in the $W^{1,1}$-norm for the variance.

Figures 2 and 3 show the estimated errors of the solution's first moment on the left hand side and of the solution's second moment on the right hand side, each versus the discretisation level for the QMC and MLQMC quadrature for the two different examples. As expected, the QMC quadrature methods achieves the predicted rate of convergence in both examples, and this rate of convergence also carries over to its multilevel adaptation (MLQMC). 
Table 1 The number of samples for the first six levels and the respective parameter dimensions.

\begin{tabular}{lrrrrrr}
\hline$l$ & 0 & 1 & 2 & 3 & 4 & 5 \\
\hline$N_{l}$ & 10 & 24 & 57 & 135 & 320 & 762 \\
\hline$M_{1}$ & 17 & 26 & 30 & 36 & 44 & 52 \\
$M_{2}$ & 14 & 26 & 30 & 36 & 43 & 52 \\
\hline
\end{tabular}
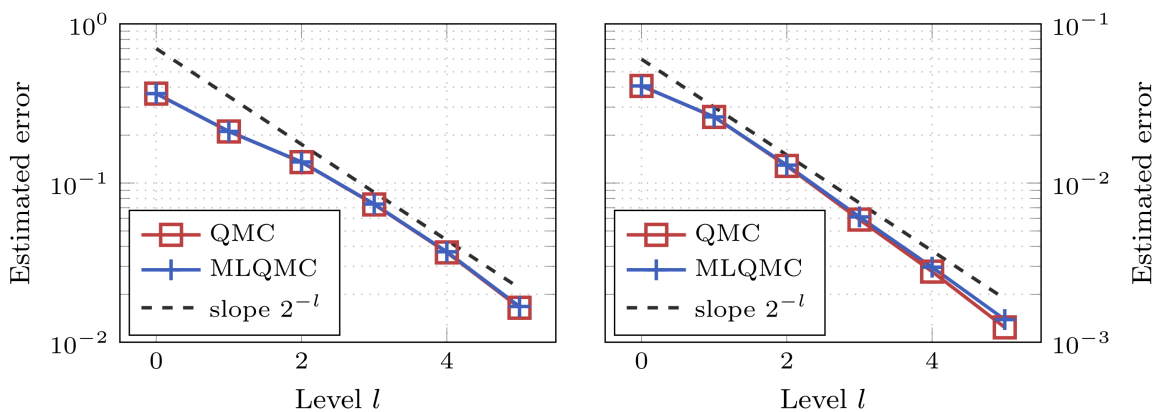

Fig. 2 Example 1. $H^{1}$-error in the $1^{\text {st }}$ moment (left) and $W^{1,1}$-error in the $2^{\text {nd }}$ moment (right).
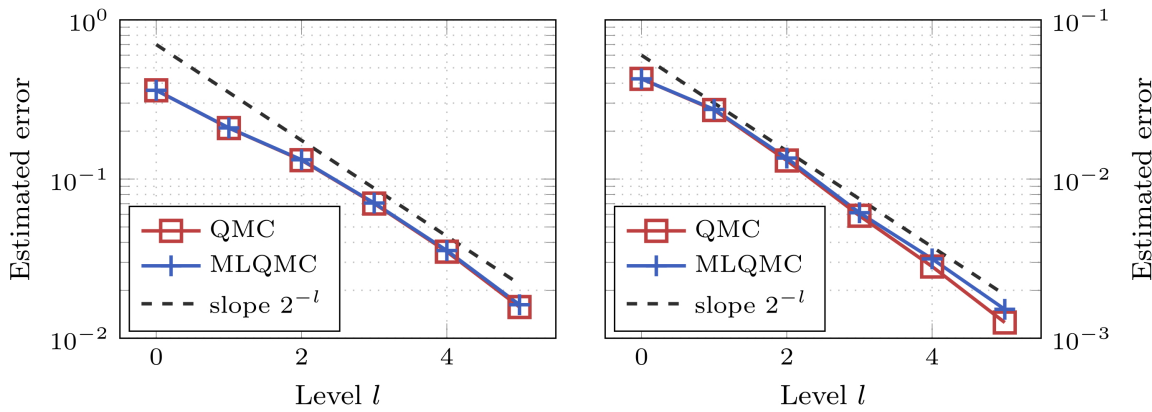

Fig. 3 Example 2. $H^{1}$-error in the $1^{\text {st }}$ moment (left) and $W^{1,1}$-error in the $2^{\text {nd }}$ moment (right).

\section{Conclusion}

In this article, we have considered the second order diffusion problem

$$
\text { for almost every } \omega \in \Omega:\left\{\begin{aligned}
-\operatorname{div}_{\mathbf{x}}\left(\mathbf{A}[\omega] \nabla_{\mathbf{x}} u[\omega]\right)=f & \text { in } D, \\
u[\omega]=0 & \text { on } \partial D,
\end{aligned}\right.
$$

with the uncertain diffusion coefficent given by

$$
\mathbf{A}[\omega]:=a \mathbf{I}+\left(\|\mathbf{V}[\omega]\|_{2}-a\right) \frac{\mathbf{V}[\omega] \mathbf{V}^{\top}[\omega]}{\mathbf{V}^{\top}[\omega] \mathbf{V}[\omega]}
$$

This models anisotropic diffusion, where the diffusion strength in the direction given by $\mathbf{V} /\|\mathbf{V}\|_{2}$ is $\|\mathbf{V}\|_{2}$ and perpendicular to it is $a$, which can be used to model both diffusion in media that consist of thin fibres or thin sheets. 
After having restated the problem in a parametric form by considering the Karhunen-Loève expansion of the random vector field $\mathbf{V}$, we have shown that, given regularity of the elliptic diffusion problem, the decay of the Karhunen-Loève expansion of $\mathbf{V}$ entirely determines the regularity of the solution's dependence on the random parameter, also when considering this higher regularity in the spatial domain.

We then leverage this result to reduce the computational complexity of the approximation of the solution's mean, by using the multilevel quasi-Monte Carlo method instead of the quasi-Monte Carlo method, while still retaining essentially the same error rate. Indeed, while in the singlelevel scheme the computational complexity is the product of the complexity of the quadrature method and of the spatial discretisation method considered, this is not the case for the multilevel scheme, which has a computational complexity that essentially scales like the complexity of the quadrature method or of the spatial discretisation method considered. The numerical experiments corroborate these theoretical findings.

While we considered the use of QMC and its multilevel adaptation, one can clearly also consider other quadrature methods, such as the anisotropic sparse grid quadrature, and then reduce the complexity by passing to their multilevel adaptations. Likewise, multilevel collocation is also applicable.

\section{References}

1. Abramowitz, M., Stegun, I.A.: Handbook of Mathematical Functions: With Formulas, Graphs, and Mathematical Tables, Appl. Math. Ser., vol. 55. Dover Publications, N. Chemsford, MA (1964)

2. Barth, A., Schwab, C., Zollinger, N.: Multi-level Monte Carlo finite element method for elliptic PDEs with stochastic coefficients. Numer. Math. 119(1), 123-161 (2011)

3. Bayer, J.D., Blake, R.C., Plank, G., Trayanova, N.A.: A novel rule-based algorithm for assigning myocardial fiber orientation to computational heart models. Ann. Biomed. Eng. 40(10), 2243-2254 (2012)

4. Beck, J., Tempone, R., Nobile, F., Tamellini, L.: On the optimal polynomial approximation of stochastic PDEs by Galerkin and collocation methods. Math. Models Methods Appl. Sci. 22(9), $1250023(2012)$

5. Braess, D.: Finite Elemente. Theorie, schnelle Löser und Anwendungen in der Elastizitätstheorie., 5th edn. Springer, Berlin (2013)

6. Brenner, S.C., Scott, L.R.: The Mathematical Theory of Finite Element Methods, 3rd edn. Springer, New York (2008)

7. Băcuţă, C., Li, H., Nistor, V.: Differential operators on domains with conical points: precise uniform regularity estimates. Rev. Roumaine de Math. Pures Appl. 62(3), 383-411 (2017)

8. Cliffe, K.A., Giles, M.B., Scheichl, R., Teckentrup, A.L.: Multilevel Monte Carlo methods and applications to elliptic PDEs with random coefficients. Comput. Vis. Sci. 14(1), 3-15 (2011)

9. Cohen, A., DeVore, R., Schwab, C.: Convergence rates of best $N$-term Galerkin approximations for a class of elliptic sPDEs. Found. Comput. Math. 10, 615-646 (2010)

10. Constantine, G.M., Savits, T.H.: A multivariate Faà di Bruno formula with applications. Trans. Amer. Math. Soc. 248, 503-520 (1996)

11. D'Elia, M., Edwards, H.C., Hu, J., Phipps, E., Rajamanickam, S.: Ensemble grouping strategies for embedded stochastic collocation methods applied to anisotropic diffusion problems. SIAM/ASA J. Uncertain. Quantif. 6(1), 87-117 (2018)

12. Dick, J., Kuo, F.Y., Le Gia, Q.T., Nuyens, D., Schwab, C.: Higher order QMC PetrovGalerkin discretization for parametric operator equations. SIAM J. Numer. Anal. 52(6), 2676-2702 (2014)

13. Graham, I.G., Kuo, F.Y., Nichols, J.A., Scheichl, R., Schwab, C., Sloan, I.H.: Quasi-Monte Carlo finite element methods for elliptic PDEs with lognormal random coefficients. Numer. Math. 131(2), 329-368 (2015) 
14. Griebel, M., Harbrecht, H., Multerer, M.D.: Multilevel quadrature for elliptic parametric partial differential equations in case of polygonal approximations of curved domains. ArXiv e-prints arXiv:1509.09058 (2018)

15. Griebel, M., Schneider, M., Zenger, C.: A combination technique for the solution of sparse grid problems. In: P. de Groen, R. Beauwens (eds.) Iterative Methods in Linear Algebra, pp. 263-281. IMACS, Elsevier, North Holland (1992)

16. Grisvard, P.: Elliptic Problems in Nonsmooth Domains. Classics in Applied Mathematics. Society for Industrial and Applied Mathematics (2011)

17. Haji-Ali, A.L., Harbrecht, H., Peters, M., Siebenmorgen, M.: Novel results for the anisotropic sparse grid quadrature. J. Complexity 47, 62-85 (2018)

18. Halton, J.H.: On the efficiency of certain quasi-random sequences of points in evaluating multi-dimensional integrals. Numer. Math. 2(1), 84-90 (1960)

19. Harbrecht, H., Peters, M., Schneider, R.: On the low-rank approximation by the pivoted Cholesky decomposition. Appl. Numer. Math. 62, 428-440 (2012)

20. Harbrecht, H., Peters, M., Siebenmorgen, M.: On multilevel quadrature for elliptic stochastic partial differential equations. Sparse Grids and Applications 88, 161-179 (2013)

21. Harbrecht, H., Peters, M., Siebenmorgen, M.: Efficient approximation of random fields for numerical applications. Numer. Linear Algebra Appl. 22(4), 596-617 (2015)

22. Harbrecht, H., Peters, M., Siebenmorgen, M.: Analysis of the domain mapping method for elliptic diffusion problems on random domains. Numer. Math. 134(4), 823-856 (2016)

23. Harbrecht, H., Peters, M., Siebenmorgen, M.: Multilevel accelerated quadrature for PDEs with log-normal distributed random coefficient. SIAM/ASA J. Uncertain. Quantif. 4(1), 520-551 (2016)

24. Harbrecht, H., Peters, M.D., Schmidlin, M.: Uncertainty quantification for PDEs with anisotropic random diffusion. SIAM J. Numer. Anal. 55(2), 1002-1023 (2017)

25. Hille, E., Phillips, R.S.: Functional Analysis and Semi-Groups, Amer. Math. Soc. Collog. Publ., vol. 31. American Mathematical Society, Providence (1957)

26. Hoang, V.H., Schwab, C.: $N$-term Wiener chaos approximation rate for elliptic PDEs with lognormal Gaussian random inputs. Math. Models Methods Appl. Sci. 24(4), 797-826 (2014)

27. Krantz, S.G., Parks, H.R.: A Primer of Real Analytic Functions, 2nd edn. Birkhäuser Advanced Texts Basler Lehrbücher. Birkhäuser Basel (2002)

28. Kuo, F.Y., Schwab, C., Sloan, I.H.: Multi-level quasi-Monte Carlo finite element methods for a class of elliptic PDEs with random coefficients. Found. Comput. Math. 15(2), 411449 (2015)

29. Logg, A., Mardal, K.A., Wells, G.N., et al.: Automated Solution of Differential Equations by the Finite Element Method. Springer, Berlin-Heidelberg (2012)

30. Niederreiter, H.: Random Number Generation and Quasi-Monte Carlo Methods. Society for Industrial and Applied Mathematics, Philadelphia, PA (1992)

31. Rodríguez-Cantano, R., Sundnes, J., Rognes, M.E.: Uncertainty in cardiac myofiber orientation and stiffnesses dominate the variability of left ventricle deformation response. Int. J. Numer. Meth. Biomed. Eng. 0(0), e3178 (2019)

32. Rohmer, D., Sitek, A., Gullberg, G.T.: Reconstruction and visualization of fiber and laminar structure in the normal human heart from ex vivo diffusion tensor magnetic resonance imaging (dtmri) data. Investigat. Radiol. 42(11), 777-789 (2007)

33. Sermesant, M., et al.: Patient-specific electromechanical models of the heart for the prediction of pacing acute effects in CRT: A preliminary clinical validation. Med. Image Analy. 16(1), 201-215 (2012)

34. Sobol', I.M.: Distribution of points in a cube and approximate evaluation of integrals. Zh. Vychisl. Mat. Mat. Fiz. 7, 784-802 (1967)

35. Teckentrup, A.L., Jantsch, P., Webster, C.G., Gunzburger, M.: A multilevel stochastic collocation method for partial differential equations with random input data. SIAM/ASA J. Uncertain. Quantif. 3(1), 1046-1074 (2015)

36. Wang, X.: A constructive approach to strong tractability using quasi-Monte Carlo algorithms. J. Complexity 18, 683-701 (2002) 